\title{
Distribuzioni di tensione per intagli soggetti a torsione in condizioni elastiche ed elastoplastiche
}

\author{
Michele Zappalorto, Paolo Lazzarin \\ Dipartimento di Tecnica e Gestione dei Sistemi Industriali, Università di Padova, stradella San Nicola, 3-36100, Vicenza, \\ Italia, email: rappalorto@gest.unipd.it, plazzarin@gest.unipd.it
}

RiASSUNTO. Il lavoro riporta delle soluzioni analitiche in forma chiusa per le distribuzioni di tensione generate $\mathrm{da}$ intagli circonferenziali in componenti assialsimmetrici soggetti a torsione, in condizioni lineari elastiche ed elastoplastiche. Il problema teorico in condizioni lineari elastiche è stato impostato e risolto utilizzando la teoria dei potenziali nel dominio complesso e una serie di opportuni sistemi di riferimento in coordinate curvilinee, evitando l'uso di mappature conformi. Le soluzioni proposte hanno un ampio range di applicabilità, in termini di dimensioni e forma dell'intaglio e di diametro dell'albero. Il problema elastoplastico è stato invece risolto utilizzando la tecnica delle trasformazioni odografiche, al fine di rendere lineari le equazioni nonlineari fondamentali del problema.

Il contributo rappresenta la sintesi di una serie di lavori più ampi a cura degli stessi autori.

ABSTRaCT. Closed form solutions for the elastic and elastic-plastic stress fields created by circumferential notches in an axisymmetric shaft under torsional loading are developed. The linear elastic boundary value problem has been formulated by an approach using complex potential functions and some curvilinear coordinate systems. The solutions obtained for the shear stresses have a wide range of applicability, both in terms of the size and shape of the notches and the diameter of the shafts. Conversely the elastic-plastic problem has been solved in closed-form by using the hodograph transformation technique, which reduces the non-linear governing equations into a linear equation system.

The present paper is a synthesis of some contributions recently published the same authors.

Parole ChIAve. Intagli, distribuzioni di tensione elastiche e elastoplastiche, NSIFs, densità di energia di deformazione (SED).

\section{INTRODUZIONE}

L a conoscenza delle distribuzioni lineari elastiche delle tensioni nelle adiacenze di intagli è di grande importanza nella valutazione della resistenza a fatica ad alto numero di cicli di componenti strutturali. Il contributo più famoso allo studio analitico di alberi indeboliti da intagli raccordati circonferenziali soggetti a torsione è dovuto a Neuber [1], il quale determinò in modo sistematico il fattore teorico di concentrazione delle tensioni $K_{\mathrm{t}}$ distinguendo tra intagli profondi e poco profondi (deep e shallow notches). Le analisi di Neuber si basavano sull'utilizzo combinato di un sistema di coordinate curvilinee e di una funzione di tensione tridimensionale reale. In relazione alle distribuzioni di tensione in forma chiusa, di fondamentale importanza è il lavoro di [2], i quali riuscirono a esprimere i campi di tensione nelle adiacenze di una blunt crack per i tre principali modi di sollecitazione, evidenziando analogie e differenze rispetto al caso della sharp crack. Degni di menzione sono anche alcuni recenti lavori di Seweryn e Molski [3], Qian e Hasebe [4] e Dunn et al. [5]. Questi ricercatori hanno fornito le distribuzioni di tensione generate da intagli a $\mathrm{V}$ non raccordati in presenza di sollecitazioni di taglio antiplanare. Per le tensioni è stata sempre utilizzata una formulazione a variabili separate.

Uno degli obiettivi del presente lavoro è quello di fornire delle espressioni in forma chiusa per le distribuzioni di tensione e deformazione indotte da intagli circonferenziali di forma semi-ellittica, parabolica o iperbolica in alberi assialsimmetrici soggetti a torsione. Il problema matematico è stato formalizzato utilizzando la teoria dei potenziali nel dominio complesso 
in combinazione con due opportuni sistemi di riferimento in coordinate curvilinee, evitando così l'uso di mappature conformi. E' messo in evidenza analiticamente il legame esistente tra le distribuzioni delle tensioni e i principali parametri geometrici dell'intaglio (come raggio di raccordo e angolo di apertura).

Le soluzioni proposte hanno carattere generale e un ampio range di applicabilità, consentendo di trattare intagli di forma anche molto differente semplicemente variando il valore dei parametri geometrici significativi. Tali soluzioni si riconducono, in alcuni casi particolari, ad alcune soluzioni classiche già riportate nella letteratura precedente (cricca, blunt crack, intaglio a $\mathrm{V}$ non raccordato).

Quando il raggio di raccordo all'apice dell'intaglio è ridotto, il livello di tensione all'apice diviene molto alto, superando il limite di snervamento e inducendo quindi una zona plastica all'apice dell'intaglio di dimensioni paragonabili a quella della zona di processo che controlla il meccanismo di frattura. In tali circostanze, la conoscenza dell'influenza indotta dalla zona plastica localizzata sulle distribuzioni di tensione vicino a cricche o intagli è di fondamentale importanza nella valutazione dell'affidabilità in servizio dei componenti meccanici. Negli ultimi anni in letteratura è stata posta una grande attenzione alla determinazione dei campi di tensione e deformazione a modo I, II e III nelle adiacenze di cricche o intagli a V a spigolo vivo in presenza di plasticità. Di fondamentale importanza sono i lavori di Hutchinson [6,7] e Rice e Rosengren [8] che fornirono una soluzione elastoplastica per i campi asintotici di tensione indotti da una cricca sollecitata a modo I. Nei decenni successivi, notevole attenzione è stata posta agli effetti di eventuali termini di ordine superiore sulle distribuzioni di tensione in presenza di sollecitazioni sia di modo I, sia di modo II [9]; le analisi sono state inoltre estese agli intagli a spigolo vivo con angolo di apertura diverso da zero [10] per i quali è stato discusso anche il caso di modo misto (I+II) in presenza di ampi angoli di apertura [11]. In questi lavori il materiale è modellato secondo la teoria $\mathrm{J}_{2} \mathrm{e}$ le funzioni angolari sono determinate numericamente (tipicamente con tecniche di multi-shooting). Il problema nonlineare antiplanare può invece essere risolto in forma chiusa con l'ausilio di mappature conformi che permettono di linearizzare le equazioni differenziali che governano il problema $[12,13]$.

Parallelamente, numerosi lavori in letteratura sono stati dedicati alla determinazione delle tensioni e delle deformazioni elastoplastiche all'apice di intagli raccordati. Tra questi sicuramente il più famoso è il lavoro di Neuber [14], il quale analizzò un corpo prismatico con due intagli raccordati simmetrici in condizioni di taglio antiplanare e ottenne una soluzione secondo la quale la media geometrica dei fattori di concentrazione delle tensioni e delle deformazioni è uguale al fattore teorico di concentrazione delle tensioni, per una qualsiasi legge costitutiva che lega le tensioni alle deformazioni. La 'regola di Neuber' è basata sull'ipotesi che il legame esistente tra la tensione reale all'apice dell'intaglio e la tensione nominale possa essere rappresentato con una particolare funzione definita da Neuber "Leading function". Come possibile alternativa alla regola di Neuber, Molski e Glinka [15] formalizzarono il criterio dell'equivalenza della densità di energia di deformazione considerando componenti con intagli raccordati soggetti a trazione o flessione, in presenza di plasticità localizzata. Quel criterio è anche stato recente riformulato per intagli a spigolo vivo, considerando la costanza dell'energia di deformazione su un volume finito di materiale centrato sull'apice dell'intaglio [16].

Tuttavia, in letteratura non esiste ancora una soluzione in forma chiusa per le distribuzioni di tensione nella zona plastica all'apice di intagli raccordati, né esiste un modello analitico che fornisca una transizione graduale tra meccanica della frattura e meccanica dell'intaglio elastoplastiche in funzione del raggio di raccordo. Il presente lavoro, focalizzato su questi temi, considera un intaglio parabolico soggetto a taglio antiplanare e un materiale elastico perfettamente plastico o elastico incrudente con legge di potenza. Più precisamente gli obiettivi del lavoro possono essere così riassunti:

- Fornire un frame comune per l'analisi dei campi di tensione e deformazione indotti da cricche o intagli parabolici in condizioni di taglio antiplanare e un legame analitico tra SIF plastici ed SIF elastici (così come ottenuti da un'analisi lineare elastica);

- Esprimere analiticamente la variazione della SED all'apice dell'intaglio o su un volume finito che circonda l'apice di una cricca rispetto al caso lineare elastico.

Il presente contributo rappresenta la sintesi di una serie di lavori più ampi a cura degli stessi autori [17-20].

\section{INTAGLI SOGGETTI A TORSIONE IN REGIME LINEARE ELASTICO}

\section{Preliminari matematici}

i consideri un corpo assialsimmetrico indebolito da un intaglio di forma generica, costituito da materiale isotropo e

S omogeneo. Si consideri inoltre un sistema di riferimento cartesiano $(x, y, z)$ con l'origine ad un'opportuna distanza dall'apice dell'intaglio (Fig. 1). Sia tale corpo sollecitato da una tensione nominale di taglio $\tau$, la quale genera solamente uno spostamento $w$ in direzione $z$, normale al piano $(x, y)$ dell'intaglio. In queste condizioni valgono per le tensioni, le deformazioni e lo spostamento $w$ le seguenti relazioni: 


$$
\tau_{\mathrm{zx}}-\mathrm{i} \tau_{\mathrm{zy}}=\mathrm{H}^{\prime}(\mathrm{z}) \quad \gamma_{\mathrm{zx}}-\mathrm{i} \gamma_{\mathrm{zy}}=\frac{\mathrm{H}^{\prime}(\mathrm{z})}{\mathrm{G}} \quad \mathrm{w}=\frac{\operatorname{Re}\{\mathrm{H}(\mathrm{z})\}}{\mathrm{G}}
$$

In queste relazioni, la funzione $H(z)$ è una funzione olomorfa di forma arbitraria e variabile da caso a caso a seconda delle condizioni al contorno del problema.

Si noti come le tensioni e gli spostamenti non siano influenzati dall'origine del sistema di riferimento, e ciò rende le espressioni $(1 \mathrm{a}-\mathrm{c})$ indipendenti da tale scelta. E' infine opportuno osservare come il simbolo " $₹$ " venga utilizzato in questo lavoro per indicare sia la variabile complessa $z=x+i y$, sia la coordinata cartesiana antiplanare mostrata in Fig. 1.

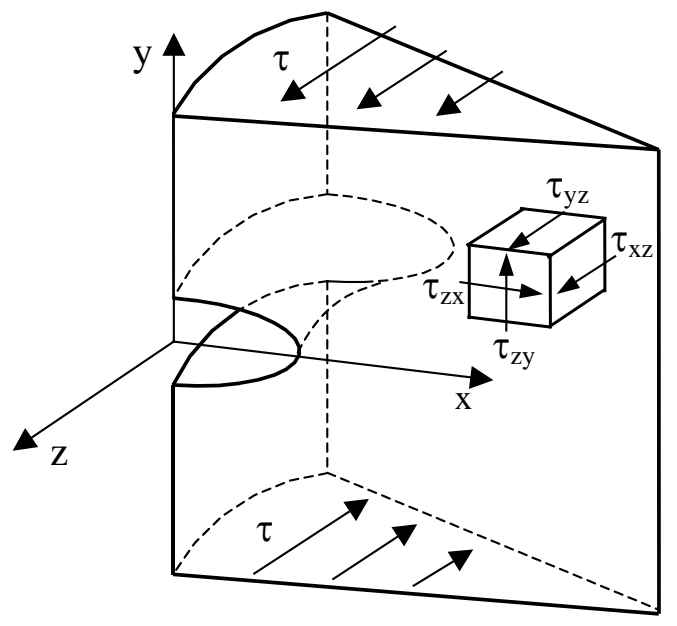

Figura 1: Componente assialsimmetrico indebolito da un intaglio circonferenziale e soggetto a taglio antiplanare.

\section{Intagli di forma semiellittica}

Considerazioni di carattere generale. Il problema relativo ad intagli di forma ellittica può essere affrontato utilizzando il sistema di coordinate curvilinee generato dalla trasformazione (Fig. 2):

$$
\mathrm{z}=\cosh \zeta
$$

dove $c$ è una costante e $z=x+i y$ e $\zeta=\xi+i \eta$ sono le variabili complesse rispettivamente nel piano fisico e nel piano trasformato.

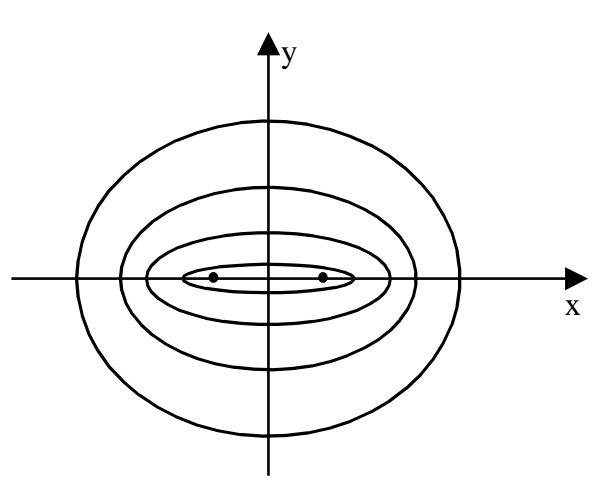

(a)

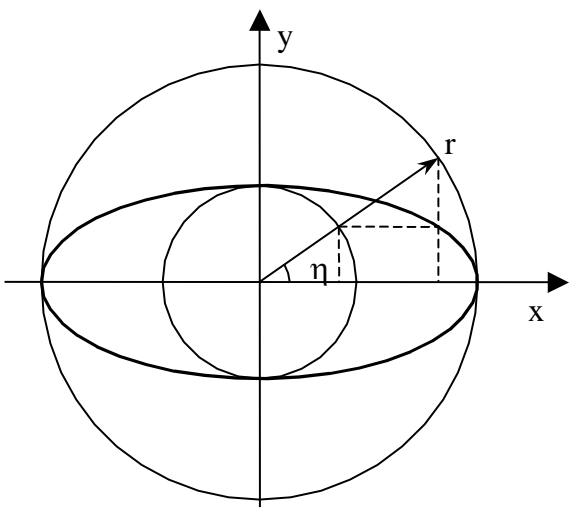

(b)

Figura 2: (a) famiglia di ellissi con gli stessi fuochi; (b) costruzione parametrica dell'ellisse.

Differenti valori di $\xi$ danno origine a una famiglia di ellissi tutte caratterizzate dagli stessi fuochi, posizionati a $x= \pm c$. Fissato $\xi=\xi_{0}$ e variando $\eta$, si ottiene una particolare ellisse appartenente alla famiglia confocale, di semiassi maggiore e minore rispettivamente pari a $a=c \cosh \xi_{0}$ e $b=c \sinh \xi_{0}$. 
L’equazione (2) può essere invertita per $\mathrm{x}, \mathrm{y}>0$. Si ottiene:

$$
\xi=\ln \sqrt{\left(x+A \cos \frac{\beta}{2}\right)^{2}+\left(y+A \sin \frac{\beta}{2}\right)^{2}}-\ln c, \quad \eta=\arcsin \left(\frac{y}{c \sinh \xi}\right)
$$

e ciò permette, una volta note le coordinate fisiche di un punto nel piano $(x, y)$, di determinare i valori corrispondenti delle variabili trasformate. I parametri $A$ e $\beta$ sono forniti in forma chiusa nel riferimento [17].

Si consideri un intaglio circonferenziale semiellittico in un albero assialsimmetrico intagliato e soggetto a torsione. Il potenziale che utilizzeremo per ottenere la soluzione presenta la seguente forma:

$$
\mathrm{H}(\mathrm{z})=\mathrm{Ac} \cosh \zeta+\mathrm{B} \operatorname{csinh} \zeta
$$

Quindi, poichè: $\frac{\partial z}{\partial \zeta}=c \sinh \zeta$, è possibile scrivere:

$$
\begin{aligned}
\mathrm{H}^{\prime}(\mathrm{z}) & =\frac{\partial \mathrm{H}(\mathrm{z})}{\partial \zeta} \cdot \frac{\partial \zeta}{\partial \mathrm{z}}=\left(\mathrm{A}_{1}+\mathrm{iA}_{2}\right)+\left(\mathrm{B}_{1}+\mathrm{iB}_{2}\right) \operatorname{coth} \zeta \\
& =\left(\mathrm{A}_{1}+\mathrm{B}_{1} \frac{\sinh 2 \xi}{\cosh 2 \xi-\cos 2 \eta}+\mathrm{B}_{2} \frac{\sin 2 \eta}{\cosh 2 \xi-\cos 2 \eta}\right)+ \\
& +\mathrm{i}\left(\mathrm{A}_{2}+\mathrm{B}_{2} \frac{\sinh 2 \xi}{\cosh 2 \xi-\cos 2 \eta}-\mathrm{B}_{1} \frac{\sin 2 \eta}{\cosh 2 \xi-\cos 2 \eta}\right)
\end{aligned}
$$

L'espressione generale delle tensioni risulta quindi:

$$
\begin{aligned}
\tau_{\mathrm{zx}} & =\mathrm{A}_{1}+\mathrm{B}_{1} \frac{\sinh 2 \xi}{\cosh 2 \xi-\cos 2 \eta}+\mathrm{B}_{2} \frac{\sin 2 \eta}{\cosh 2 \xi-\cos 2 \eta} \\
\tau_{\mathrm{zy}} & =-\mathrm{A}_{2}-\mathrm{B}_{2} \frac{\sinh 2 \xi}{\cosh 2 \xi-\cos 2 \eta}+\mathrm{B}_{1} \frac{\sin 2 \eta}{\cosh 2 \xi-\cos 2 \eta}
\end{aligned}
$$

Condirioni al contorno per un albero di sezione infinita. Se l'intaglio ha dimensioni infinite, le condizioni al contorno possono essere espresse nella seguente forma:

- Se $|z| \rightarrow \infty, \tau_{\mathrm{zy}}=\tau$ e $\tau_{\mathrm{zx}}=0$, dove $\tau$ è la tensione di taglio nominale;

- Sul bordo dell'intaglio $\left(\xi=\xi_{0}\right), \tau_{\mathrm{z} \xi}=0$. Quando $\left(\xi=\xi_{0}\right)$ e $\left(\eta=\frac{\pi}{2}\right)$ allora $\tau_{\mathrm{zy}}=\tau_{\mathrm{z} \xi}$;

- Quando $\eta=\frac{\pi}{2}, \tau_{\mathrm{zx}}=0$.

Sostituendo le condizioni al contorno nelle Eq. 6 si ottengono i coefficienti $A_{i}$ e $B_{i}$ e le tensioni risultano quindi:

$$
\left\{\begin{array}{l}
\tau_{\mathrm{zy}}=\frac{\tau}{\mathrm{a}-\mathrm{b}}\left(\mathrm{a} \frac{\sinh 2 \xi}{\cosh 2 \xi-\cos 2 \eta}-\mathrm{b}\right) \\
\tau_{\mathrm{zx}}=-\frac{\tau \mathrm{a}}{\mathrm{a}-\mathrm{b}} \frac{\sin 2 \eta}{\cosh 2 \xi-\cos 2 \eta}
\end{array}\right.
$$

All'apice dell'intaglio le Eq. 7 danno:

$$
\left.\tau_{\mathrm{zy}}\right|_{\substack{\eta=0 \\ \xi=\xi_{0}}}=\frac{\tau}{\mathrm{a}-\mathrm{b}}\left(\mathrm{a} \frac{\sinh 2 \xi_{0}}{\cosh 2 \xi_{0}-1}-\mathrm{b}\right)=\tau\left(1+\frac{\mathrm{a}}{\mathrm{b}}\right)
$$


e quindi il fattore teorico di concentrazione delle tensioni risulta $K_{t}=1+a / b$, in accordo con Neuber [1].

La soluzione ottenuta, che risulta matematicamente esatta solo nel caso di un albero di diametro infinito, può tuttavia essere applicata anche ad alberi di dimensione finita, almeno fino a quando il rapporto $\mathrm{a} / \mathrm{R}$ è inferiore a 0.05 , con errori nella determinazione della tensione massima inferiori al 10\%.

Per fare ciò è però necessario tenere in considerazione l'andamento decrescente lineare della tensione nominale nella sezione, semplicemente con l'aggiunta alle espressioni delle tensioni di un fattore correttivo:

$$
\left\{\begin{array}{c}
\tau_{\mathrm{zy}} \\
\tau_{\mathrm{zx}}
\end{array}\right\}=\left\{\begin{array}{l}
\frac{\tau}{\mathrm{a}-\mathrm{b}}\left(\mathrm{a} \frac{\sinh 2 \xi}{\cosh 2 \xi-\cos 2 \eta}-\mathrm{b}\right) \\
\frac{-\tau \mathrm{a}}{\mathrm{a}-\mathrm{b}}\left(\frac{\sin 2 \eta}{\cosh 2 \xi-\cos 2 \eta}\right)
\end{array}\right\}\left(1-\frac{\mathrm{c} \cos \eta\left(\cosh \xi-\cosh \xi_{0}\right)}{\mathrm{R}+\left(\mathrm{a}-\mathrm{c} \cos \eta \cosh \xi_{0}\right)}\right)
$$

Le Fig. 3 e 4 mostrano un confronto tra i risultati analitici e i risultati di alcune analisi agli elementi finiti condotte su alberi in cui la dimensione dell'intaglio è molto inferiore rispetto al raggio netto dell'albero. L'accordo appare molto soddisfacente.

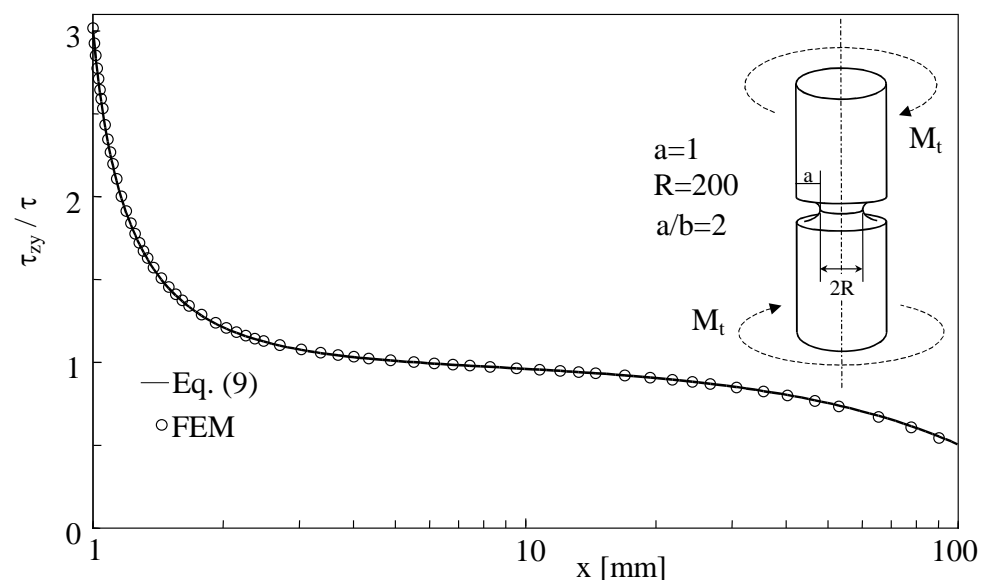

Figura 3: Componente di tensione $\tau_{\mathrm{zy}}$ lungo la bisettrice geometrica dell'intaglio.

Le tensioni sono normalizzate rispetto alla tensione nominale.

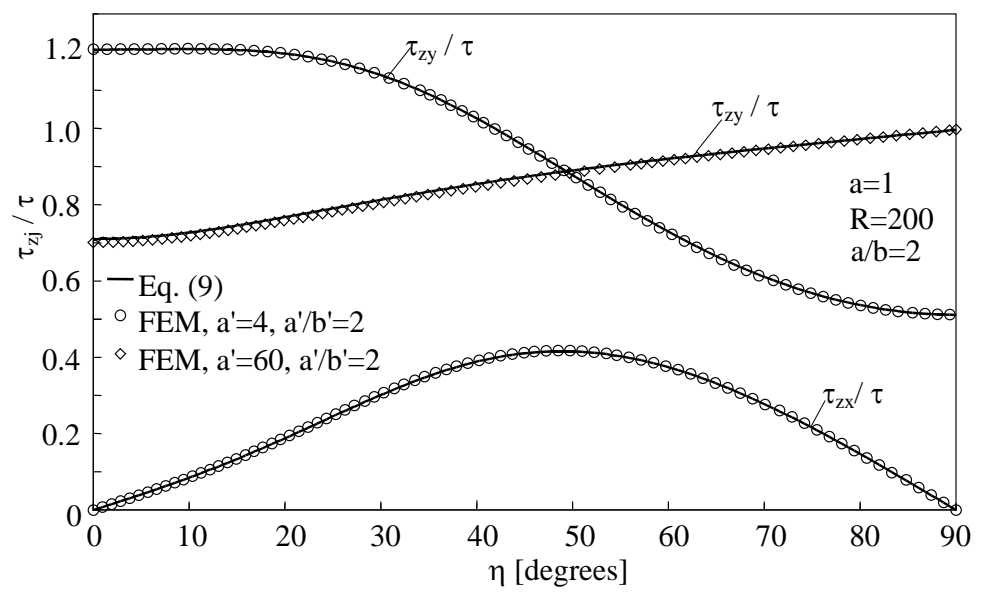

Figura 4: Componenti di tensione lungo due percorsi ellittici centrati nell'origine del sistema di riferimento (x,y); $\mathrm{a}^{\prime}=4 \mathrm{~mm}$ e $\mathrm{a}^{\prime}=60 \mathrm{~mm}$, mentre $\mathrm{a}^{\prime} / \mathrm{b}^{\prime}=2$. Le tensioni sono normalizzate rispetto alla tensione nominale.

Cricca circonferenziale su albero infinito. La cricca circonferenziale può essere trattata da un punto di vista matematico come un intaglio semiellittico in cui il semiasse minore b tende a zero. Utilizzando quindi i risultati della precedente sessione per intagli semiellittici, è possibile scrivere: 


$$
\tau \frac{\mathrm{z}}{\sqrt{\mathrm{z}^{2}-\mathrm{a}^{2}}}=\tau_{\mathrm{zy}}+\mathrm{i} \tau_{\mathrm{zx}}
$$

dove a è la lunghezza della cricca. Si noti come l'espressione posta alla sinistra nell'equazione (10) coincida esattamente con la funzione di tensione di Westergaard per il modo III.

Dall'equazione (10) è quindi agevole determinare dapprima l'espressione del fattore di intensificazione delle tensioni:

$$
\mathrm{K}_{\mathrm{III}}=\left.\lim _{\mathrm{x} \rightarrow \mathrm{a}} \sqrt{2 \pi(\mathrm{x}-\mathrm{a})} \tau_{\mathrm{zy}}\right|_{\mathrm{y}=0}=\tau \sqrt{\pi \mathrm{a}}
$$

e quindi l'espressione delle tensioni in un intorno dell'apice della cricca:

$$
\left\{\begin{array}{c}
\tau_{\mathrm{zx}} \\
\tau_{\mathrm{zy}}
\end{array}\right\}=\frac{\tau \sqrt{\mathrm{a}}}{\sqrt{2 \mathrm{r}}}\left\{\begin{array}{c}
-\sin \left(\frac{\varphi}{2}\right) \\
\cos \left(\frac{\varphi}{2}\right)
\end{array}\right\}=\frac{\mathrm{K}_{\mathrm{III}}}{\sqrt{2 \pi \mathrm{r}}}\left\{\begin{array}{c}
-\sin \left(\frac{\varphi}{2}\right) \\
\cos \left(\frac{\varphi}{2}\right)
\end{array}\right\}
$$

Intaglio semicircolare su albero infinito. In linea di principio il sistema di coordinate ellittiche utilizzato nella soluzione precedente non è più valido nel caso di intaglio circolare, quando $\mathrm{a}=\mathrm{b}(\mathrm{c}=0)$, che comporta una discontinuità matematica nella definizione del sistema in questione. Nonostante ciò si può notare che la soluzione precedente continua a essere valida anche quando il rapporto a/b è molto vicino a 1 , permettendo quindi di trattare l'intaglio semicircolare come il limite per $(a / b) \rightarrow 1$. Quest'idea è confermata da analisi agli elementi finiti condotte su alberi quasi infiniti $(\mathrm{a} / \mathrm{R}=0.005)$ indeboliti da intagli semiellittici con $a / b=1.001$, come mostrato in Fig. 5.

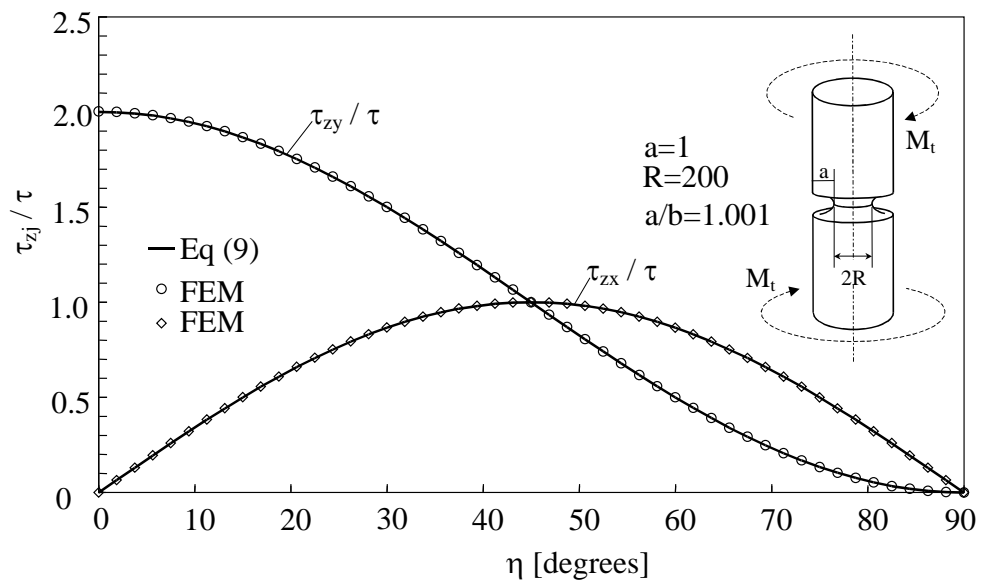

Figura 5: Componenti di tensione $\tau_{\mathrm{zy}}$ and $\tau_{\mathrm{zx}}$ sul bordo dell'intaglio nel caso di un intaglio che può essere considerato semicircolare

$$
(\mathrm{a} / \mathrm{b}=1.001) \text {. Le tensioni sono normalizzate rispetto alla tensione nominale. }
$$

Condizioni al contorno per un albero di sežione finita. Come prima approssimazione nel caso di alberi intagliati a diametro finito è possibile mantenere per il potenziale complesso la medesima forma, e modificare in modo opportuno solo le condizioni al contorno; infatti le condizioni poste nella precedente trattazione all'infinito non risultano più valide. Il nuovo sistema di condizioni al contorno risulta quindi:

$$
\begin{aligned}
& -\left.\tau_{\mathrm{zx}}\right|_{\mathrm{\eta}=\frac{\pi}{2}}=\mathrm{A}_{1}+\mathrm{B}_{1} \frac{\sinh 2 \xi}{\cosh 2 \xi+1}=0
\end{aligned}
$$

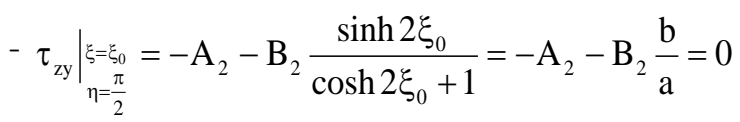




$$
-\left.\tau_{\mathrm{zy}}\right|_{\substack{\xi=\xi_{0} \\ \eta=0}}=-\mathrm{A}_{2}-\mathrm{B}_{2} \frac{\sinh 2 \xi_{0}}{\cosh 2 \xi_{0}-1}=-\mathrm{A}_{2}-\mathrm{B}_{2} \frac{\mathrm{a}}{\mathrm{b}}=\tau_{\max }
$$

Sostituendo, come prima, tali condizioni all'interno delle espressioni generali delle tensioni, Eq. 6 , è possibile ottenere $\mathrm{i}$ valori dei coefficienti $A_{i}$ e $B_{i}$ e quindi le espressioni finali delle tensioni:

$$
\left\{\begin{array}{l}
\tau_{z y}=\frac{\tau}{a-b} \frac{K_{t, \text { gross }}}{1+\frac{a}{b}}\left(a \frac{\sinh 2 \xi}{\cosh 2 \xi-\cos 2 \eta}-b\right)\left(1-\frac{\mathrm{c} \cos \eta\left(\cosh \xi-\cosh \xi_{0}\right)}{R+\left(a-\cos \eta \cosh \xi_{0}\right)}\right) \\
\tau_{z x}=\frac{-\tau a}{a-b} \frac{K_{t, g r o s s}}{1+\frac{a}{b}} \frac{\sin 2 \eta}{\cosh 2 \xi-\cos 2 \eta}\left(1-\frac{\mathrm{c} \cos \eta\left(\cosh \xi-\cosh \xi_{0}\right)}{R+\left(a-\cos \eta \cosh \xi_{0}\right)}\right)
\end{array}\right.
$$

Particolarmente utile è la tensione $\tau_{\mathrm{zy}}$ lungo la bisettrice dell'intaglio:

$$
\tau_{\mathrm{zy}}=\frac{\mathrm{b} \tau_{\max }}{\mathrm{a}^{2}-\mathrm{b}^{2}}\left(\frac{\mathrm{ax}}{\sqrt{\mathrm{x}^{2}-\mathrm{c}^{2}}}-\mathrm{b}\right) \cdot\left(1-\frac{(\mathrm{x}-\mathrm{a})}{\mathrm{R}}\right)
$$

che permette, tra l'altro, di determinare un'espressione approssimata del fattore teorico di concentrazione delle tensioni grazie a un'equazione di equilibrio alle rotazioni sulla sezione netta, mettendo in gioco il contributo fornito dalla tensione nominale, che varia da un valore massimo $\tau^{*}$ a zero, e la componente di taglio generata dall'intaglio, $\tau_{\mathrm{zy}}$.

Effettuato quindi il cambio di variabile $t=R+a-x$, è possibile imporre l'equilibrio come:

$$
\int_{0}^{2 \pi} \int_{0}^{\mathrm{R}} \tau^{*} \frac{\mathrm{t}^{3}}{\mathrm{R}} \mathrm{dtd} \theta=\int_{0}^{2 \pi} \int_{0}^{\mathrm{R}} \tau_{\mathrm{zy}} \mathrm{t}^{2} \mathrm{dtd} \theta
$$

che fornisce:

$$
\mathrm{K}_{\mathrm{t}, \text { net }}=\frac{\mathrm{C}^{2}}{\mathrm{~b}} \cdot \frac{1}{\mathrm{C}\left(\frac{\mathrm{a}}{\mathrm{R}}, \frac{\mathrm{a}}{\mathrm{b}}\right)} ; \quad \mathrm{K}_{\mathrm{t}, \text { gross }}=\mathrm{K}_{\mathrm{t}, \text { net }} \cdot\left(\frac{\mathrm{R}_{\mathrm{g}}}{\mathrm{R}}\right)^{3}
$$

essendo:

$$
\begin{aligned}
& \mathrm{C}\left(\frac{\mathrm{a}}{\mathrm{R}}, \frac{\mathrm{a}}{\mathrm{b}}\right)=\left\{\frac { \mathrm { a } } { 2 } \cdot \operatorname { l n } ( \frac { \frac { \mathrm { b } } { \mathrm { R } } - \frac { \mathrm { a } } { \mathrm { R } } } { \sqrt { ( 1 + \frac { \mathrm { a } } { \mathrm { R } } ) ^ { 2 } - ( \frac { \mathrm { c } } { \mathrm { R } } ) ^ { 2 } } - ( 1 + \frac { \mathrm { a } } { \mathrm { R } } ) } ) \left[( ( 1 + \frac { \mathrm { a } } { \mathrm { R } } ) ^ { 2 } - ( \frac { \mathrm { c } } { \mathrm { R } } ) ^ { 2 } ) \left(18\left(1+\frac{\mathrm{a}}{\mathrm{R}}\right)^{2}-\right.\right.\right. \\
& \left.\left.3\left(\left(1+\frac{\mathrm{a}}{\mathrm{R}}\right)^{2}-\left(\frac{\mathrm{c}}{\mathrm{R}}\right)^{2}\right)\right)-15\left(1+\frac{\mathrm{a}}{\mathrm{R}}\right)^{4}\right]+\frac{\mathrm{ab}}{2 \mathrm{R}} \cdot\left[13\left(1+\frac{\mathrm{a}}{\mathrm{R}}\right)\left(\left(1+\frac{\mathrm{a}}{\mathrm{R}}\right)^{2}-\left(\frac{\mathrm{c}}{\mathrm{R}}\right)^{2}\right)-15\left(1+\frac{\mathrm{a}}{\mathrm{R}}\right)^{3}+\right. \\
& \left.3\left(\left(1+\frac{\mathrm{a}}{\mathrm{R}}\right)^{2}-\left(\frac{\mathrm{c}}{\mathrm{R}}\right)^{2}\right)-5\left(1+\frac{\mathrm{a}}{\mathrm{R}}\right)^{2}-2\left(1+\frac{\mathrm{a}}{\mathrm{R}}\right)-2\right]- \\
& \left.\frac{\mathrm{a}}{2} \sqrt{\left(1+\frac{\mathrm{a}}{\mathrm{R}}\right)^{2}-\left(\frac{\mathrm{c}}{\mathrm{R}}\right)^{2}}\left[13\left(1+\frac{\mathrm{a}}{\mathrm{R}}\right)\left(\left(1+\frac{\mathrm{a}}{\mathrm{R}}\right)^{2}-\left(\frac{\mathrm{c}}{\mathrm{R}}\right)^{2}\right)-15\left(1+\frac{\mathrm{a}}{\mathrm{R}}\right)^{3}\right]-\mathrm{b}\right\}
\end{aligned}
$$

\section{Intagli di forma parabolica e iperbolica}

Una prima classe di soluzioni. Consideriamo un sistema di coordinate iperboliche generate dalla seguente trasformazione [21, 22]: 


$$
z=c \cosh \zeta
$$

dove $c$ è una costante e $z=x+i y$ e $\zeta=\xi+i \eta$ sono le variabili complesse nel piano fisico e nel piano trasformato.

Differenti valori di $\eta$ danno origine a una famiglia di iperboli tutte caratterizzate dagli stessi fuochi, posizionati alla distanza $x= \pm$ c. Fissato $\eta=\eta_{0}$ e variando $\xi$, si ottiene una particolare iperbole appartenente alla famiglia confocale, di costanti pari a $a=c \cos \eta_{0}$ e $b=c \sin \eta_{0}$ (vedi Fig. 6).

L’equazione (20) può essere invertita per $(x, y)>0$. Si ottiene:

$$
\begin{aligned}
& \eta=\arctan \left(\frac{\mathrm{y}+\mathrm{A} \sin \frac{\beta}{2}}{\mathrm{x}+\mathrm{Acos} \frac{\beta}{2}}\right) \\
& \xi=\operatorname{arcsinh}\left(\frac{\mathrm{y}}{\mathrm{csin} \eta}\right)
\end{aligned}
$$

e ciò permette, una volta note le coordinate fisiche di un punto nel piano $(x, y)$, di determinare i valori corrispondenti delle variabili trasformate. I parametri $A$ e $\beta$ sono riportati in forma chiusa in [19].

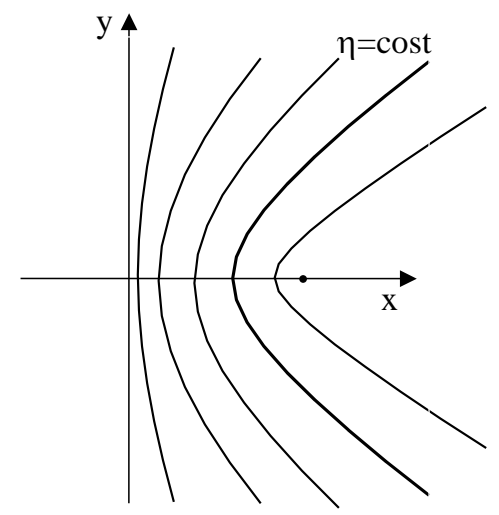

(a)

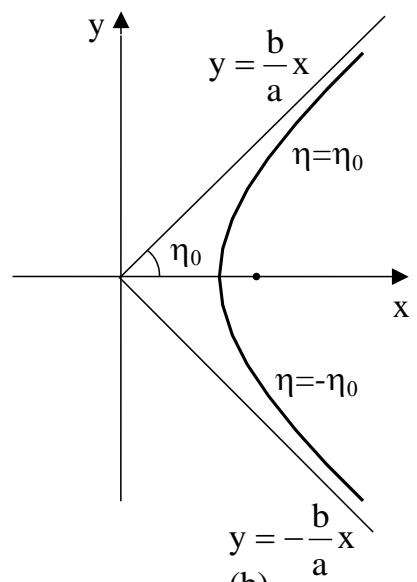

(b)

Figura 6: Famiglia di iperboli confocali (a); profilo iperbolico (primo e quarto quadrante) (b).

Il problema di un intaglio iperbolico in un corpo infinito può essere affrontato osservando che la tensione nominale sulla sezione lorda deve essere nulla in modo tale da garantire una tensione nominale finita sulla sezione netta. Un potenziale che soddisfa automaticamente questa condizione è il seguente:

$$
H(z)=\left(A_{1}+i A_{2}\right) c \zeta
$$

Poiché: $\frac{\partial z}{\partial \zeta}=\operatorname{csinh} \zeta$, è possibile scrivere:

$$
\begin{aligned}
\mathrm{H}^{\prime}(\mathrm{z}) & =\frac{\partial \mathrm{H}(\mathrm{z})}{\partial \zeta} \cdot \frac{\partial \zeta}{\partial \mathrm{z}}=\frac{\left(\mathrm{A}_{1}+\mathrm{iA}_{2}\right)}{\sinh \zeta}= \\
& \left(\frac{2 \mathrm{~A}_{1} \cos \eta \sinh \xi+2 \mathrm{~A}_{2} \sin \eta \cosh \xi}{\cosh 2 \xi-\cos 2 \eta}\right)+\mathrm{i}\left(\frac{2 \mathrm{~A}_{2} \cos \eta \sinh \xi-2 \mathrm{~A}_{1} \sin \eta \cosh \xi}{\cosh 2 \xi-\cos 2 \eta}\right)
\end{aligned}
$$

L'espressione generale delle tensioni risulta quindi: 


$$
\begin{aligned}
\tau_{\mathrm{zx}} & =\frac{2 \mathrm{~A}_{1} \cos \eta \sinh \xi+2 \mathrm{~A}_{2} \sin \eta \cosh \xi}{\cosh 2 \xi-\cos 2 \eta} \\
\tau_{\mathrm{zy}} & =-\frac{2 \mathrm{~A}_{2} \cos \eta \sinh \xi-2 \mathrm{~A}_{1} \sin \eta \cosh \xi}{\cosh 2 \xi-\cos 2 \eta}
\end{aligned}
$$

Imponendo le seguenti condizioni al contorno:

$$
\left.\tau_{\mathrm{zx}}\right|_{\substack{\eta=\eta_{0} \\ \xi=0}}=\frac{2 \mathrm{~A}_{2} \sin \eta_{0}}{1-\cos 2 \eta_{0}}=0 \rightarrow \mathrm{A}_{2}=0, \tau_{\max }=\frac{\mathrm{cA}_{1}}{\sqrt{\mathrm{c}^{2}-\mathrm{a}^{2}}} \rightarrow \mathrm{A}_{1}=\tau^{*} \frac{\mathrm{K}_{\mathrm{t}, \mathrm{net}}}{\sqrt{1+\frac{\mathrm{a}}{\rho}}}
$$

le distribuzioni di tensione assumono la seguente forma:

$$
\begin{aligned}
\tau_{\mathrm{zx}} & =\tau^{*} \frac{2 \mathrm{~K}_{\mathrm{t}, \mathrm{net}}}{\sqrt{1+\frac{\mathrm{a}}{\rho}}} \frac{\cos \eta \sinh \xi}{\cosh 2 \xi-\cos 2 \eta} \\
\tau_{\mathrm{zy}} & =\tau^{*} \frac{2 \mathrm{~K}_{\mathrm{t}, \mathrm{net}}}{\sqrt{1+\frac{\mathrm{a}}{\rho}}} \frac{\sin \eta \cosh \xi}{\cosh 2 \xi-\cos 2 \eta}
\end{aligned}
$$

La soluzione ottenuta, che risulta matematicamente esatta solo nel caso di taglio antiplanare uniforme, può tuttavia essere applicata anche ad alberi soggetti a torsione; è però necessario tenere in considerazione l'andamento decrescente lineare della tensione nominale nella sezione, semplicemente con l'aggiunta alle espressioni delle tensioni di un fattore correttivo:

$$
\begin{aligned}
\tau_{\mathrm{zx}} & =\tau^{*} \frac{\mathrm{K}_{\mathrm{t}, \mathrm{net}}}{\sqrt{1+\frac{\mathrm{a}}{\rho}}} \cdot \frac{\cos ^{2} \eta \sinh 2 \xi}{\cos \eta_{0} \cosh \xi^{*}(\cosh 2 \xi-\cos 2 \eta)} \\
\tau_{\mathrm{zy}} & =\tau^{*} \frac{\mathrm{K}_{\mathrm{t}, \mathrm{net}}}{\sqrt{1+\frac{\mathrm{a}}{\rho}}} \cdot \frac{\sin 2 \eta \cosh ^{2} \xi}{\cos \eta_{0} \cosh \xi^{*}(\cosh 2 \xi-\cos 2 \eta)}
\end{aligned}
$$

L'espressione del fattore teorico di concentrazione delle tensioni può, a questo punto, essere ottenuto con un'equazione di equilibrio sulla sezione netta:

$$
\int_{\mathrm{A}} \tau(\mathrm{x}) \mathrm{xdA}=\int_{\mathrm{A}} \tau_{\mathrm{zy}} \mathrm{xdA}
$$

che fornisce:

$$
\mathrm{K}_{\mathrm{t}, \text { net }}=\frac{3}{4} \frac{\left(1+\sqrt{\frac{\mathrm{a}}{\rho}+1}\right)^{2}}{\left(1+2 \sqrt{\frac{\mathrm{a}}{\rho}+1}\right)}
$$

in accordo con Neuber [1].

Le Figure 7 e 8 mostrano un confronto tra i risultati analitici e quelli di alcune analisi agli elementi finiti condotte su alberi in cui la dimensione dell'intaglio è molto superiore rispetto al raggio netto dell'albero (intaglio "deep"); l'accordo appare molto soddisfacente. 


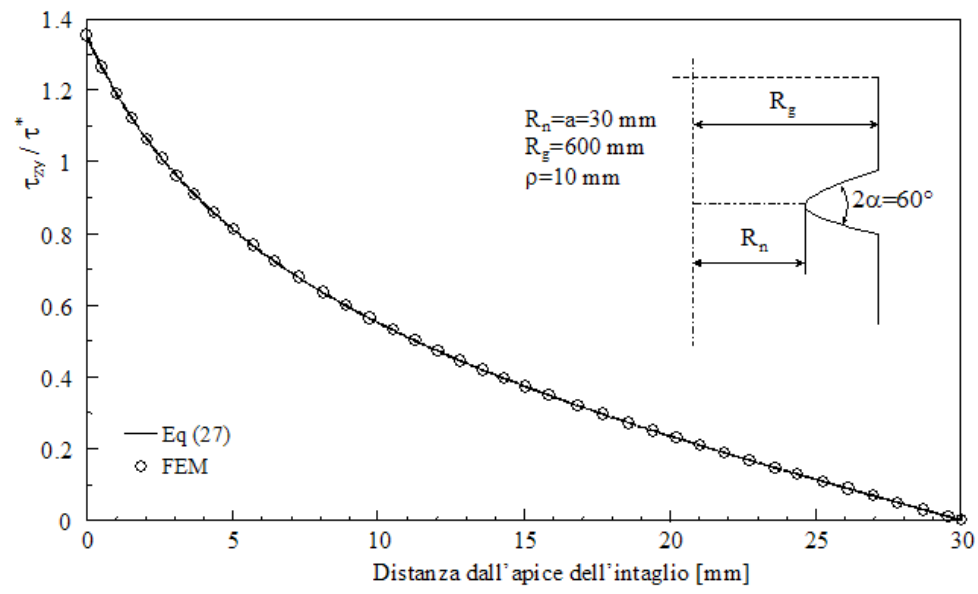

Figura 7: Componente di tensione $\tau_{z y}$ lungo la bisettrice dell'intaglio normalizzata rispetto alla tensione nominale sull'area netta.

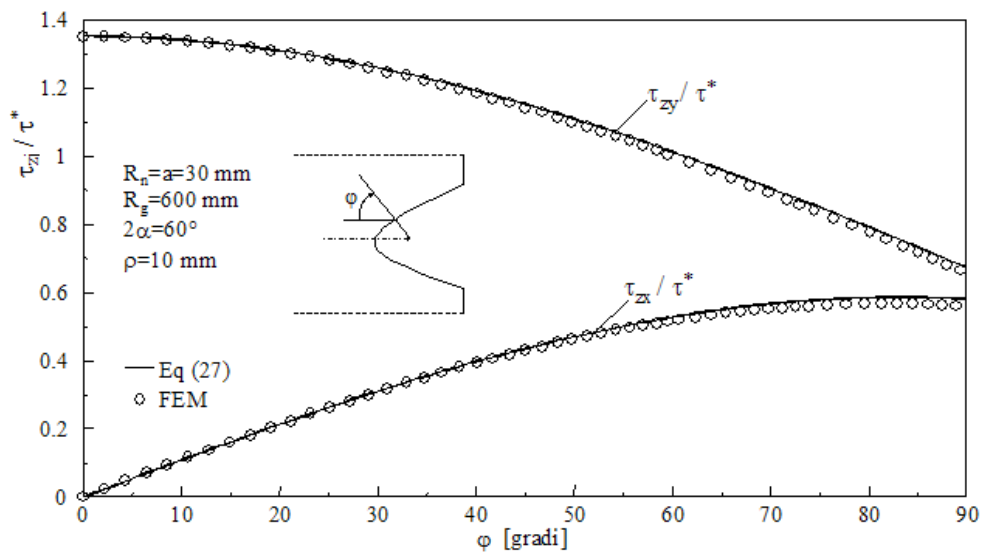

Figura 8: Campi di tensione lungo il bordo dell'intaglio normalizzati rispetto alla tensione nominale sulla sezione netta.

Una seconda classe di solurioni. Consideriamo ora il sistema di coordinate curvilinee generato dalla trasformazione [1]:

$$
\mathrm{Z}=\mathrm{w}^{\mathrm{q}}
$$

dove $z=x+i y$ e $w=u+i v$ sono le variabili complesse nel piano fisico e nel piano trasformato e $q$ è un numero reale funzione dell'angolo di apertura $2 \alpha$ :

$$
\mathrm{q}=\frac{2 \pi-2 \alpha}{\pi}=\frac{2 \gamma}{\pi}
$$

L'equazione (30) può essere riscritta nella forma seguente:

$$
\left\{\begin{array}{l}
\mathrm{u}=\mathrm{r}^{\frac{1}{\mathrm{q}}} \cos \frac{\varphi}{\mathrm{q}}, \quad \mathrm{r}=\left(\mathrm{u}^{2}+\mathrm{v}^{2}\right)^{\frac{\mathrm{q}}{2}} \\
\mathrm{v}=\mathrm{r}^{\frac{1}{\mathrm{q}}} \sin \frac{\varphi}{\mathrm{q}}
\end{array}\right.
$$

Il sistema di coordinate curvilinee introdotto permette di descrivere intagli parabolici $(q=2)$ o iperbolici $(1<\mathrm{q}<2)$. La generica curva caratterizzata dal valore $\boldsymbol{u}=\mu_{0}$ interseca l'asse delle ascisse al valore $r_{0}$, che dipende oltre che dall'angolo di apertura, anche dal raggio di raccordo per mezzo dell'espressione $r_{0}=\rho(q-1) / q$ (Fig. 9).

Il problema matematico può essere risolto utilizzando lo stesso potenziale adottato per la trasformazione precedente: 
$H^{\prime}(z)=\frac{\left(A_{1}+i A_{2}\right)}{q(u+i v)^{q-1}}=\frac{\left(A_{1}+i A_{2}\right)(u-i v)^{q-1}}{q\left(u^{2}+v^{2}\right)^{q-1}}$.

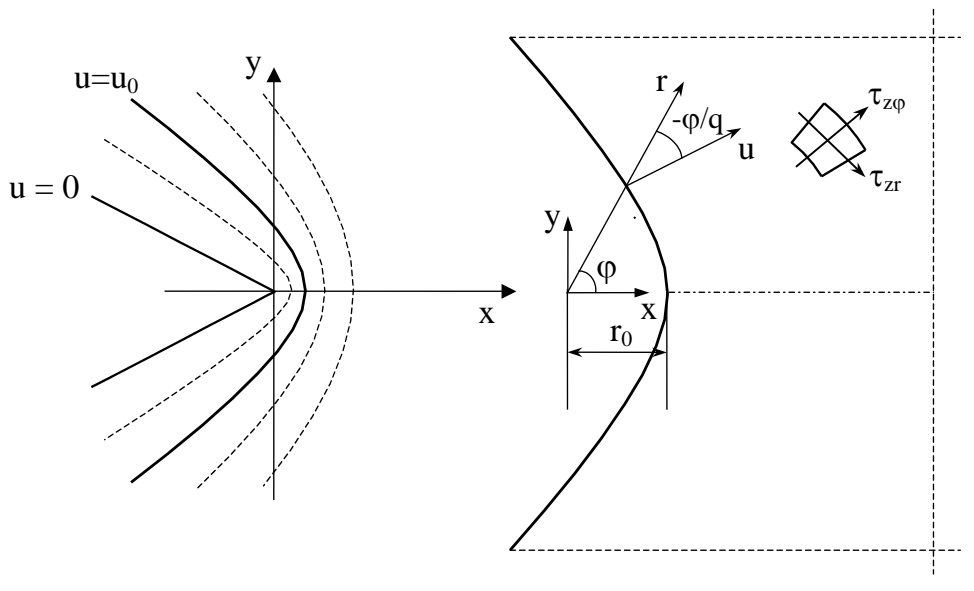

(a)

(b)

Figura 9: (a) Sistema di coordinate curvilinee $(u, v)$;

(b) sistema di riferimento adottato per la soluzione.

Posta la seguente condizione al contorno:

$$
\left.\tau_{\mathrm{zx}}\right|_{\mathrm{v}=0 \mathrm{u}} ^{\mathrm{u}=0} \underset{\mathrm{qu}_{0}}{\mathrm{q}-1}=0 \rightarrow \mathrm{A}_{1}=0
$$

si ottiene:

$$
\left\{\begin{array} { l } 
{ \tau _ { \mathrm { zx } } = \frac { \mathrm { A } _ { 2 } } { \mathrm { qr } ^ { \frac { \mathrm { q } - 1 } { \mathrm { q } } } } \operatorname { s i n } ( \frac { \mathrm { q } - 1 } { \mathrm { q } } \varphi ) } \\
{ \tau _ { \mathrm { zy } } = \frac { - \mathrm { A } _ { 2 } } { \mathrm { qr } ^ { \frac { \mathrm { q } - 1 } { \mathrm { q } } } } \operatorname { c o s } ( \frac { \mathrm { q } - 1 } { \mathrm { q } } \varphi ) }
\end{array} \left\{\begin{array}{l}
\tau_{\mathrm{zr}}=\frac{-\mathrm{A}_{2}}{\mathrm{qr}^{\frac{\mathrm{q}-1}{\mathrm{q}}}} \sin \left(\frac{1}{\mathrm{q}} \varphi\right) \\
\tau_{\mathrm{z} \varphi}=\frac{-\mathrm{A}_{2}}{\mathrm{qr}^{\frac{\mathrm{q}-1}{\mathrm{q}}}} \cos \left(\frac{1}{\mathrm{q}} \varphi\right)
\end{array}\right.\right.
$$

Indicando con $\lambda_{3}=1 / q$, è possibile determinare la costante $A_{2}$ in funzione della tensione di taglio massima o del fattore generalizzato di intensificazione delle tensioni di modo III:

$$
A_{2}=-\frac{K_{3 \rho}}{\sqrt{2 \pi} \lambda_{3}}, \quad A_{2}=\frac{-\tau_{\max }}{\lambda_{3} r_{0}^{\lambda_{3}-1}}
$$

dove

$$
\mathrm{K}_{3 \rho}=\sqrt{2 \pi} \lim _{\mathrm{r} \rightarrow \mathrm{r}_{\mathrm{o}}^{+}}\left[\mathrm{r}^{1-\lambda_{3}} \tau_{\mathrm{z \varphi}}(\mathrm{r}, \varphi=0)\right]
$$

Le tensioni, espresse in coordinate polari risultano quindi:

$$
\left\{\begin{array}{c}
\tau_{\mathrm{zr}}(\mathrm{r}, \varphi) \\
\tau_{\mathrm{z} \varphi}(\mathrm{r}, \varphi)
\end{array}\right\}=\tau_{\max }\left(\frac{\mathrm{r}}{\mathrm{r}_{0}}\right)^{\lambda_{3}-1}\left\{\begin{array}{c}
\sin \lambda_{3} \varphi \\
\cos \lambda_{3} \varphi
\end{array}\right\}
$$


o in modo equivalente:

$$
\left\{\begin{array}{c}
\tau_{\mathrm{zr}}(\mathrm{r}, \varphi) \\
\tau_{\mathrm{z} \varphi}(\mathrm{r}, \varphi)
\end{array}\right\}=\frac{\mathrm{K}_{3 \rho} \mathrm{r}^{\lambda_{3}-1}}{\sqrt{2 \pi}}\left\{\begin{array}{c}
\sin \lambda_{3} \varphi \\
\cos \lambda_{3} \varphi
\end{array}\right\}
$$

Uguagliando le due espressioni si ottiene la seguente relazione fondamentale che lega NSIF generalizzato e tensione massima:

$$
\mathrm{K}_{3 \rho}=\tau_{\max } \sqrt{2 \pi} \mathrm{r}_{0}^{1-\lambda_{3}}
$$

Si noti come nel caso di un intaglio parabolico si abbia $2 \alpha=0, \lambda_{3}=0.5$ e quindi:

$$
\mathrm{K}_{3 \rho}=\tau_{\max } \sqrt{\pi \rho}
$$

Questa relazione è stata ottenuta precedentemente da altri autori [23].

Anche in questo caso è possibile correggere le distribuzioni delle tensioni per tenere in considerazione l'effetto di decremento lineare della tensione nominale:

$$
\left\{\begin{array}{c}
\tau_{\mathrm{zr}}(\mathrm{r}, \varphi) \\
\tau_{\mathrm{z} \varphi}(\mathrm{r}, \varphi)
\end{array}\right\}=\tau_{\max } \cdot\left(1-\frac{\left(\mathrm{r}-\mathrm{r}^{\prime}\right) \cos \varphi}{\mathrm{R}^{\prime}}\right)\left(\frac{\mathrm{r}}{\mathrm{r}_{0}}\right)^{\lambda_{3}-1}\left\{\begin{array}{c}
\sin \lambda_{3} \varphi \\
\cos \lambda_{3} \varphi
\end{array}\right\} \quad \varphi \neq \pm \gamma
$$

dove

$$
r^{\prime}=\frac{r_{0}}{(\cos (\varphi / q))^{q}} \quad R^{\prime}=R+\left(r_{0}-r^{\prime} \cos \varphi\right)
$$

e $R$ è il raggio della sezione netta dell'albero.

Con un'equazione di equilibrio sulla sezione netta è inoltre possibile determinare il valore del fattore teorico di concentrazione delle tensioni per alberi soggetti a torsione indeboliti da intagli profondi (deep notches):

$$
\int_{\mathrm{A}} \tau(\mathrm{t}) \mathrm{tdA}=\left.\int_{\mathrm{A}} \tau_{\mathrm{zy}}\right|_{\mathrm{y}=0} \mathrm{tdA}
$$

ottenendo la seguente espressione:

$$
\mathrm{K}_{\mathrm{t}, \text { net }}=\frac{\mathrm{s}_{3}^{4}+10 \mathrm{~s}_{3}^{3}+35 \mathrm{~s}_{3}^{2}+50 \mathrm{~s}_{3}+24}{4 \sum_{\mathrm{j}=0}^{3} \mathrm{I}_{\mathrm{j}}}
$$

dove:

$$
\begin{aligned}
& s_{3}(2 \alpha)=\lambda_{3}-1 \\
& k\left(r_{0}, R\right)=\frac{r_{0}}{R} \\
& I_{0}=\left(1+\frac{1}{k}\right)^{s_{3}}\left[6+24 k+36 k^{2}+24 k^{3}+6 k^{4}\right] \\
& I_{1}=-k\left(24+26 s_{3}+9 s_{3}^{2}+s_{3}^{3}\right) \\
& I_{2}=-k^{2}\left(36+21 s_{3}+3 s_{3}^{2}\right) \\
& I_{3}=-k^{3}\left(24+6 s_{3}\right)-6 k^{4}
\end{aligned}
$$

Le Fig. 10 e 11 mostrano un confronto tra i risultati analitici e quelli di alcune analisi agli elementi finiti condotte su alberi indeboliti da intagli di varia forma e soggetti a torsione; l'accordo appare molto soddisfacente. 


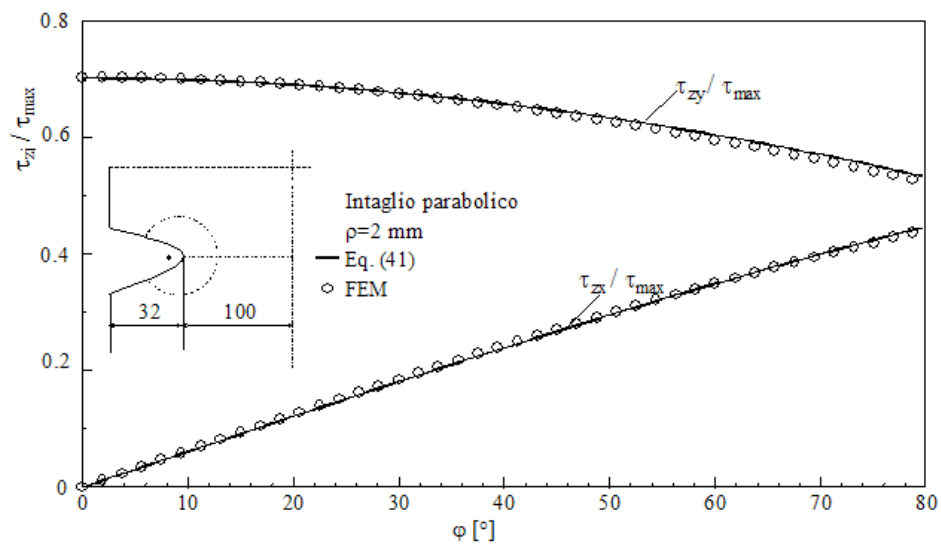

Figura 10: Campi di tensione su un percorso circolare di raggio pari a $r=2 \mathrm{~mm}$ centrato sul fuoco dell'intaglio normalizzati rispetto alla tensione massima.

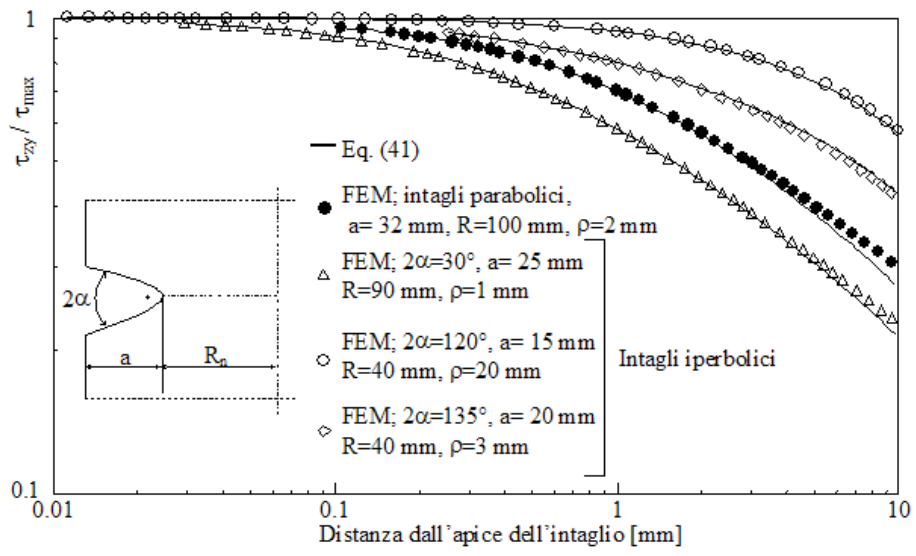

Figura 11: Componenti di tensione $\tau_{\text {zy }}$ lungo la bisettrice dell'intaglio normalizzate rispetto alla tensione massima (intagli iperbolici e parabolici).

\section{INTAGLIO PARABOLICO SOGGETTO A TAGLIO ANTIPLANARE IN REGIME ELASTOPLASTICO}

\section{Materiale a comportamento elastico perfettamente plastico}

onsideriamo un intaglio parabolico in un materiale elastico perfettamente plastico e soggetto a taglio antiplanare.

Utilizzando un approccio già adottato per la cricca (da Irwin, [24], a Unger, [25]), il confine elastoplastico $\Omega$ può

essere determinato introducendo le distribuzioni di tensioni lineari elastiche nella seguente condizione di snervamento:

$$
\tau_{\mathrm{zx}}^{2}+\tau_{\mathrm{zy}}^{2}=\tau_{0}^{2}
$$

ottenendo per il raggio plastico la seguente espressione:

$$
\left.\mathrm{r}\right|_{\Omega}=\mathrm{R}_{\mathrm{pz}}=\frac{1}{2 \pi}\left(\frac{\mathrm{K}_{3 \rho}^{\mathrm{e}}}{\tau_{0}}\right)^{2}
$$

La funzione di tensione $\phi$ per lo sforzo antiplanare può essere legata alle componenti di tensione per mezzo delle seguenti espressioni: 


$$
\tau_{\mathrm{zx}}=\frac{\partial \phi}{\partial \mathrm{y}} \quad \tau_{\mathrm{zy}}=-\frac{\partial \phi}{\partial \mathrm{x}}
$$

Introducendo $\phi$ nell'equazione (46) e chiamando $p=\partial \phi / \partial x$ and $q=\partial \phi / \partial y$ si ottiene:

$$
\mathrm{p}^{2}+\mathrm{q}^{2}=\tau_{0}^{2}
$$

L'equazione (49) può essere convertita in un sistema di equazioni differenziali ordinarie utilizzando il metodo di CharpitLagrange [25-27]. Il sistema risulta:

$$
\left\{\begin{array}{c}
\frac{\partial \mathrm{x}}{\partial \mathrm{s}}=\frac{\partial \mathrm{F}}{\partial \mathrm{p}}=2 \mathrm{p} \\
\frac{\partial \mathrm{y}}{\partial \mathrm{s}}=\frac{\partial \mathrm{F}}{\partial \mathrm{q}}=2 \mathrm{q} \\
\frac{\partial \mathrm{p}}{\partial \mathrm{s}}=-\left(\frac{\partial \mathrm{F}}{\partial \mathrm{x}}+\mathrm{p} \frac{\partial \mathrm{F}}{\partial \phi}\right)=0 \\
\frac{\partial \mathrm{q}}{\partial \mathrm{s}}=-\left(\frac{\partial \mathrm{F}}{\partial \mathrm{y}}+\mathrm{q} \frac{\partial \mathrm{F}}{\partial \phi}\right)=0
\end{array}\right.
$$

dove $s$ è una variabile ausiliaria. Integrando rispetto a $s$ e utilizzando i valori iniziali per $s=0$, cioè quando $r=R_{\mathrm{pz}}$, è possibile determinare le costanti $\iota_{\mathrm{i}}$ e quindi le variabili $x, y, p, q$ :

$$
\begin{aligned}
& x=-2 \tau_{0} \sqrt{1-t^{2}} s+R_{p z}\left(1-2 t^{2}\right) \\
& y=-2 \tau_{0} t s+2 R_{p z} t \sqrt{1-t^{2}} \\
& p=-\tau_{0} \sqrt{1-t^{2}} \\
& q=-\tau_{0} t
\end{aligned}
$$

laddove $t=\sin \frac{\varphi}{2}$.

Il parametro ausiliario t può ora essere determinato in funzione delle coordinate fisiche $x$ e $y$ :

$$
t=\frac{y}{\sqrt{\left(x+R_{p z}\right)^{2}+y^{2}}}
$$

Quindi, le espressioni finali per le tensioni nella zona plastica risultano:

$$
\begin{aligned}
& \tau_{\mathrm{zx}}=-\tau_{0} \frac{\mathrm{y}}{\sqrt{\left(\mathrm{x}+\mathrm{R}_{\mathrm{pz}}\right)^{2}+\mathrm{y}^{2}}} \\
& \tau_{\mathrm{zy}}=\tau_{0} \frac{\mathrm{x}+\mathrm{R}_{\mathrm{pz}}}{\sqrt{\left(\mathrm{x}+\mathrm{R}_{\mathrm{pz}}\right)^{2}+\mathrm{y}^{2}}}
\end{aligned}
$$

Definiamo ora un nuovo sistema di riferimento con origine $\mathrm{O}^{\prime}$, traslato di $\mathrm{R}_{\mathrm{pz}}$ nella direzione $x$ (vedi Fig. 12). Le coordinate rispetto a $\mathrm{O}^{\prime}$ risultano quindi:

$$
\overline{\mathrm{r}}=\sqrt{\left(\mathrm{x}+\mathrm{R}_{\mathrm{pz}}\right)^{2}+\mathrm{y}^{2}} \quad \bar{\varphi}=\arctan \left(\frac{\mathrm{y}}{\mathrm{x}+\mathrm{R}_{\mathrm{pz}}}\right)
$$


Il confine elastoplastico continua a essere centrato nell'origine $\mathrm{O}$, ma le componenti di tensione valutate rispetto al nuovo sistema di riferimento diventano:

$$
\begin{aligned}
\tau_{\mathrm{zx}} & =-\tau_{0} \sin \bar{\varphi} \\
\tau_{\mathrm{zy}} & =\tau_{0} \cos \bar{\varphi}
\end{aligned}
$$

che costituisce una soluzione ammissibile in accordo con la teoria delle slip lines di Sokolovskii [28]. L'entità della traslazione, $R_{\mathrm{pz}}$, dipende dall'NSIF elastico e dalla tensione di snervamento $\tau_{0}$.

L'estensione della zona plastica di fronte all'intaglio risulta quindi:

$$
\Delta \mathrm{P}=2 \mathrm{R}_{\mathrm{pz}}-\frac{\rho}{2}
$$

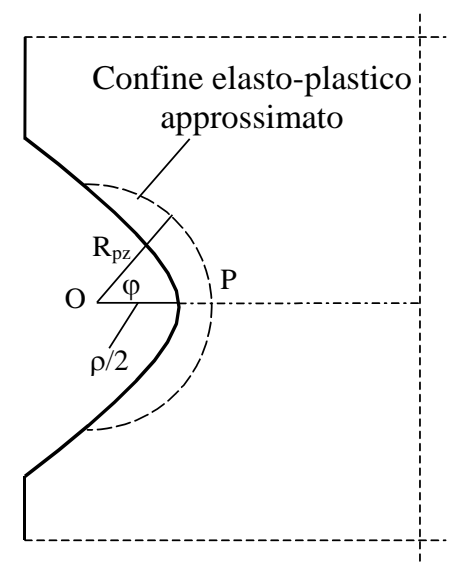

(a)

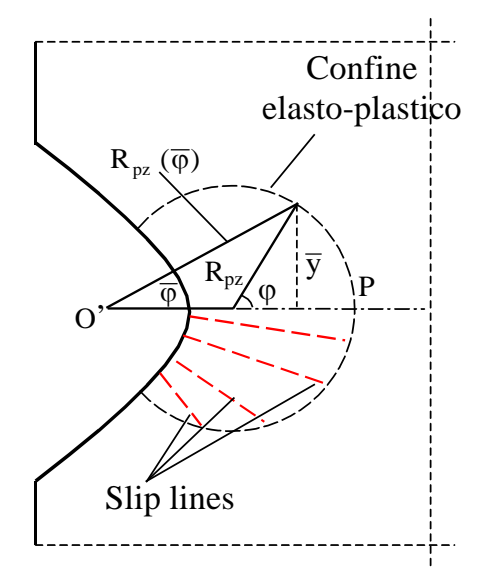

(b)

Figura 12: Confronto tra la zona plastica approssimata (a), basata sulla soluzione lineare elastica, e la zona plastica esatta (b) per un materiale a comportamento elastico perfettamente plastico.

Quando l'intaglio diviene una cricca $(\rho=0)$, si ottiene $\Delta P=2 R_{\mathrm{pz}}$ e l'Eq.56a risulta in accordo con la soluzione di Hult e McClintock [12, 25, 29].

Si osservi inoltre come due intagli con lo stesso valore di $K_{3 \rho}^{\mathrm{e}}$, ma con due differenti raggi di raccordo, presentano due differenti dimensioni della zona plastica; in particolare minore è il raggio di raccordo, maggiore è la zona plastica. Si noti inoltre come sostituendo nell'equazione (56a) l'espressione di $R_{\mathrm{pz}}$ e $K_{3 \rho}^{\mathrm{e}}=\tau_{\max }^{\mathrm{e}} \sqrt{\pi \rho}$ sia possibile ottenere:

$$
\Delta \mathrm{P}=\rho\left[\left(\frac{\tau_{\max }^{\mathrm{e}}}{\tau_{0}}\right)^{2}-\frac{1}{2}\right]
$$

La relazione mostra che, a parità di tensione massima, la zona plastica è tanto minore quanto minore è il raggio di raccordo.

Lo spostamento $w$ per un intaglio parabolico in condizioni lineari elastiche risulta pari a [19]:

$$
\mathrm{W}=\sqrt{\frac{2 \mathrm{r}}{\pi}} \frac{\mathrm{K}_{3 \rho}^{\mathrm{e}}}{\mathrm{G}} \sin \frac{\varphi}{2}
$$

Quindi sostituendo $r=R_{\mathrm{pz}}$, si ottiene:

$$
\mathrm{w}_{\mathrm{p}}=\left.\mathrm{w}\right|_{\Omega}=\sqrt{\frac{2 \mathrm{R}_{\mathrm{pz}}}{\pi}} \frac{\mathrm{K}_{3 \rho}^{\mathrm{e}}}{\mathrm{G}} \sin \frac{\varphi}{2}=\frac{\left(\mathrm{K}_{3 \rho}^{\mathrm{e}}\right)^{2}}{\pi \mathrm{G} \tau_{0}} \sin \bar{\varphi}
$$


Contemporaneamente, l'equazione di Hencky:

$$
\tau_{\mathrm{zy}} \frac{\partial \mathrm{w}}{\partial \mathrm{x}}-\tau_{\mathrm{zx}} \frac{\partial \mathrm{w}}{\partial \mathrm{y}}=0
$$

assicura che $w=$ const lungo le slip lines [28], e quindi Eq. (23) può essere estesa all'intera zona plastica.

Gli scorrimenti, in coordinate cartesiane, risultano quindi:

$$
\begin{aligned}
& \gamma_{\mathrm{zx}}=\frac{\partial \mathrm{w}}{\partial \bar{\varphi}} \cdot \frac{\partial \bar{\varphi}}{\partial \mathrm{x}}=-\frac{\left(\mathrm{K}_{3 \rho}^{\mathrm{e}}\right)^{2}}{\pi \tau_{0} \mathrm{G}} \frac{\cos \bar{\varphi} \sin \bar{\varphi}}{\overline{\mathrm{r}}} \\
& \gamma_{\mathrm{zy}}=\frac{\partial \mathrm{w}}{\partial \bar{\varphi}} \cdot \frac{\partial \bar{\varphi}}{\partial \mathrm{y}}=\frac{\left(\mathrm{K}_{3 \rho}^{\mathrm{e}}\right)^{2}}{\pi \tau_{0} \mathrm{G}} \frac{\cos ^{2} \bar{\varphi}}{\overline{\mathrm{r}}}
\end{aligned}
$$

\section{Materiale a comportamento incrudente con legge di potenza}

Trattazione matematica. Si consideri ora invece una legge lineare al di sotto dello snervamento e una legge di potenza per la zona plastica (Fig. 13):

$$
\begin{array}{ll}
\frac{\gamma}{\gamma_{0}}=\frac{\tau}{\tau_{0}} & \text { se } \tau \leq \tau_{0} \\
\frac{\gamma}{\gamma_{0}}=\left(\frac{\tau}{\tau_{0}}\right)^{n} & \text { se } \tau>\tau_{0}
\end{array}
$$

dove $\gamma_{0}=\frac{\tau_{0}}{G}$ e $1 \leq n \leq \infty$. In questa formulazione, $\gamma_{0}$ è la deformazione a snervamento e $\tau_{0}$ è la corrispondente tensione di snervamento.

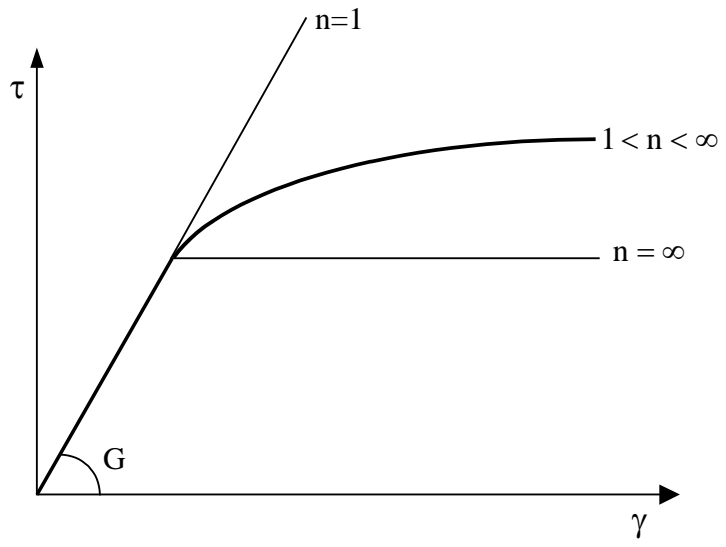

Figura 13: Curva tensioni deformazioni utilizzata per al formulazione matematica del problema.

Sono inoltre valide le seguenti relazioni:

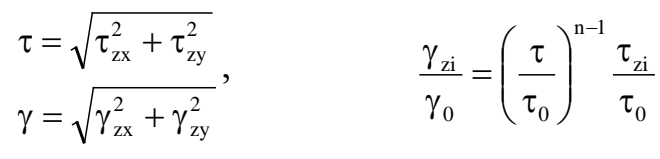

Utilizzando la trasformazione odografica suggerita da Hult and McClintock [12]:

$$
\mathrm{x}=-\frac{\partial \psi}{\partial \tau_{\mathrm{zy}}} \quad \mathrm{y}=\frac{\partial \psi}{\partial \tau_{\mathrm{zx}}}
$$


le equazioni di equilibrio e compatibilità possono essere riscritte come[13]:

$$
\frac{\partial \mathrm{x}}{\partial \tau_{\mathrm{zx}}}+\frac{\partial \mathrm{y}}{\partial \tau_{\mathrm{zy}}}=0 \quad \frac{\partial \mathrm{x}}{\partial \gamma_{\mathrm{zy}}}-\frac{\partial \mathrm{y}}{\partial \gamma_{\mathrm{zx}}}=0
$$

purchè lo Jacobiano delle trasformazioni non sia nullo.

Inoltre introducendo un sistema di coordinate polari nel piano delle tensioni:

$$
\tau_{\mathrm{zx}}=-\tau \sin \bar{\varphi} \quad \tau_{\mathrm{zy}}=\tau \cos \bar{\varphi}
$$

è possible riscrivere le coordinate $(\mathrm{x}, \mathrm{y})$ nella seguente forma:

$$
\begin{aligned}
& \mathrm{x}=-\frac{\partial \psi}{\partial \tau_{\mathrm{zy}}}=-\frac{\partial \psi}{\partial \tau} \cos \bar{\varphi}+\frac{\partial \psi}{\partial \bar{\varphi}} \frac{\sin \bar{\varphi}}{\tau} \\
& \mathrm{y}=\frac{\partial \psi}{\partial \tau_{\mathrm{zx}}}=-\frac{\partial \psi}{\partial \tau} \sin \bar{\varphi}-\frac{\partial \psi}{\partial \bar{\varphi}} \frac{\cos \bar{\varphi}}{\tau}
\end{aligned}
$$

Sostituendo le Eq.66 nell'equazione di compatibilità inversa e utilizzando la legge costitutiva del materiale si ottiene:

$$
\frac{1}{\mathrm{n}} \frac{\partial^{2} \psi}{\partial \tau^{2}}+\frac{1}{\tau} \frac{\partial \psi}{\partial \tau}+\frac{1}{\tau^{2}} \frac{\partial^{2} \psi}{\partial \bar{\varphi}^{2}}=0
$$

L’Eq. 67 è un'equazione di Eulero che ammette soluzioni nella forma:

$$
\psi(\tau, \bar{\varphi})=\tau^{\mathrm{m}} \tilde{\psi}(\bar{\varphi})
$$

Sostituendo la (66) nella (65) si ottiene:

$$
\tilde{\psi}(\bar{\varphi})\left[\frac{1}{n} m(m-1)+m\right]+\tilde{\psi}^{\prime \prime}(\bar{\varphi})=0
$$

e quindi:

$$
\psi(\tau, \bar{\varphi})=\tau^{m}\left(c_{1} \sin \omega \bar{\varphi}+c_{2} \cos \omega \bar{\varphi}\right), \quad \omega=\frac{1}{n} m(m-1)+m
$$

E' possibile a questo punto risolvere il problema di Neumann, specificando il valore della derivata prima della funzione $\psi(\tau, \bar{\varphi})$ sul confine del dominio di integrazione (ovvero sul confine elastoplastico).

Se si ipotizza quindi che, in analogia con il caso elastico perfettamente plastico, l'origine del sistema di riferimento risulti traslato rispetto al centro del confine elastoplastico di una quantità pari a $k R_{\mathrm{pz}}$, con $k<1.0$, (vedi Fig. 14), dalle equazioni lineari elastiche si ottiene:

$$
\begin{gathered}
\cos \frac{\varphi}{2}=\frac{\left.\tau_{\mathrm{zy}}\right|_{\Omega}}{\frac{\mathrm{K}_{3 \rho}^{\mathrm{e}}}{\sqrt{2 \pi \mathrm{R}_{\mathrm{pz}}}}}=\frac{\left.\tau_{\mathrm{zy}}\right|_{\Omega}}{\tau_{0}} \\
\sin \frac{\varphi}{2}=-\frac{\left.\tau_{\mathrm{zx}}\right|_{\Omega}}{\frac{\mathrm{K}_{3 \rho}^{\mathrm{e}}}{\sqrt{2 \pi \mathrm{R}_{\mathrm{pz}}}}}=-\frac{\left.\tau_{\mathrm{zx}}\right|_{\Omega}}{\tau_{0}}
\end{gathered}
$$

Quindi dalle Eq. 65, 71 si ha: 


$$
\begin{aligned}
& \left.\mathrm{x}\right|_{\Omega}=\mathrm{R}_{\mathrm{pz}} \cos \varphi+\mathrm{kR}_{\mathrm{pz}}=\mathrm{R}_{\mathrm{pz}}\left(1-2 \sin ^{2} \frac{\varphi}{2}\right)+\mathrm{kR}_{\mathrm{pz}}=\mathrm{R}_{\mathrm{pz}}\left(1-2\left(\frac{\left.\tau_{\mathrm{zx}}\right|_{\Omega}}{\tau_{0}}\right)^{2}\right)+\mathrm{kR}_{\mathrm{pz}} \\
& =\mathrm{R}_{\mathrm{pz}}\left(1-2 \sin ^{2} \bar{\varphi}\right)+\mathrm{kR}_{\mathrm{pz}}=\mathrm{R}_{\mathrm{pz}} \cos 2 \bar{\varphi}+\mathrm{kR}_{\mathrm{pz}} \\
& \left.\mathrm{y}\right|_{\Omega}=\mathrm{R}_{\mathrm{pz}} \sin \varphi=2 \mathrm{R}_{\mathrm{pz}} \sin \frac{\varphi}{2} \cos \frac{\varphi}{2}=-2 \mathrm{R}_{\mathrm{pz}} \frac{\left.\tau_{\mathrm{zx}}\right|_{\Omega}}{\tau_{0}} \frac{\left.\tau_{\mathrm{zy}}\right|_{\Omega}}{\tau_{0}}=2 \mathrm{R}_{\mathrm{pz}} \sin \bar{\varphi} \cos \bar{\varphi}=\mathrm{R}_{\mathrm{pz}} \sin 2 \bar{\varphi}
\end{aligned}
$$

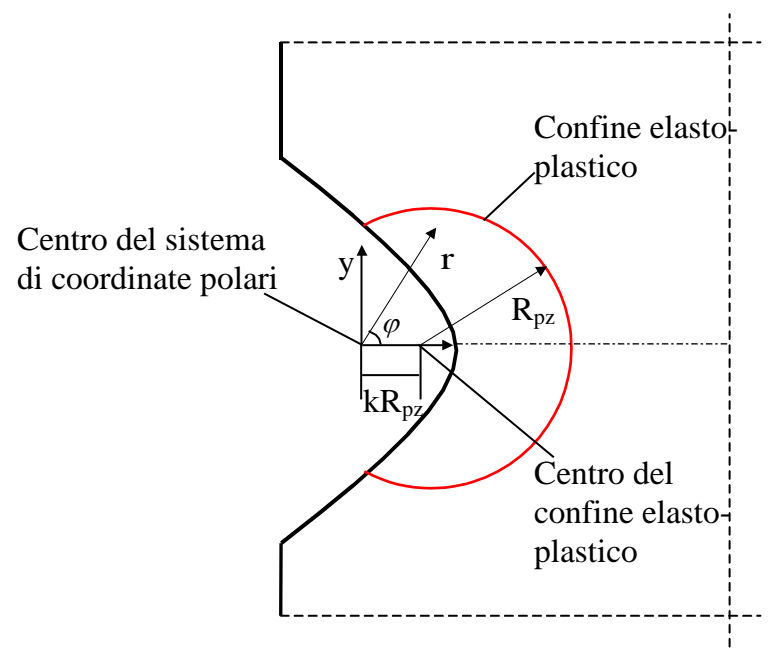

Figura 14: Zona plastica per un materiale incrudente secondo legge di potenza.

Usando l'espressione:

$$
\frac{\partial \psi}{\partial \bar{\varphi}}=\frac{\partial \psi}{\partial \tau_{\mathrm{zx}}} \frac{\partial \tau_{\mathrm{zx}}}{\partial \bar{\varphi}}+\frac{\partial \psi}{\partial \tau_{\mathrm{zy}}} \frac{\partial \tau_{\mathrm{zy}}}{\partial \bar{\varphi}}
$$

e le Eq. 61, 63, 70:

$$
\begin{aligned}
\left.\left(\frac{\partial \psi}{\partial \bar{\varphi}}\right)\right|_{\Omega} & =\left.\mathrm{x}\right|_{\Omega} \cdot \tau_{0} \sin \bar{\varphi}-\left.\mathrm{y}\right|_{\Omega} \cdot \tau_{0} \cos \bar{\varphi}=\left(\mathrm{R}_{\mathrm{pz}} \cos 2 \bar{\varphi}+\mathrm{kR} \mathrm{pz}_{0}\right) \tau_{0} \sin \bar{\varphi}-\mathrm{R}_{\mathrm{pz}} \sin 2 \bar{\varphi} \tau_{0} \cos \bar{\varphi}= \\
& =\mathrm{R}_{\mathrm{pz}} \tau_{0}(\mathrm{k}-1) \sin \bar{\varphi}
\end{aligned}
$$

In modo del tutto indipendente dall'Eq. 70 è possibile ottenere:

$$
\frac{\partial \psi(\tau, \bar{\varphi})}{\partial \bar{\varphi}}=\omega \tau^{\mathrm{m}}\left(\mathrm{c}_{1} \cos \omega \bar{\varphi}-\mathrm{c}_{2} \sin \omega \bar{\varphi}\right)
$$

Inoltre uguagliando le due formulazioni:

$$
\mathrm{c}_{1}=0, \quad \omega=1, \quad \mathrm{~m}=-\mathrm{n}, \quad \mathrm{c}_{2}=(1-\mathrm{k}) \mathrm{R}_{\mathrm{pz}} \tau_{0}^{\mathrm{n}+1}, \quad \psi=(1-\mathrm{k}) \mathrm{R}_{\mathrm{pz}} \tau_{0}^{\mathrm{n}+1} \tau^{-\mathrm{n}} \cos \bar{\varphi}
$$

e infine:

$$
\begin{aligned}
& x=\frac{(1-k) R_{p z} \tau_{0}^{n+1}}{\tau^{n+1}}\left(n \cos ^{2} \bar{\varphi}-\sin ^{2} \bar{\varphi}\right) \\
& y=\frac{(1-k) R_{p z} \tau_{0}^{n+1}}{\tau^{n+1}}(n+1) \cos \bar{\varphi} \sin \bar{\varphi}
\end{aligned}
$$


All'estremità delle zona plastica, lungo la bisettrice dell'intaglio, si ha $x_{p}=(1+k) R_{\mathrm{pz}}$ mentre $\tau=\tau_{0}$.

Quindi:

$$
\mathrm{k}=\frac{\mathrm{n}-1}{\mathrm{n}+1} \quad x_{p}=\frac{2 \mathrm{n}}{\mathrm{n}+1} \mathrm{R}_{\mathrm{pz}}
$$

A partire dalle Eq. 77a, b è possibile ottenere la coordinata radiale:

$$
\mathrm{r}=\sqrt{\mathrm{x}^{2}+\mathrm{y}^{2}}=\frac{(1-\mathrm{k}) \mathrm{R}_{\mathrm{pz}} \tau_{0}^{\mathrm{n}+1}}{\tau^{\mathrm{n}+1}} \mathrm{n} \tilde{\mathrm{F}}=\mathrm{x}_{\mathrm{p}} \frac{\tau_{0}^{\mathrm{n}+1}}{\tau^{\mathrm{n}+1}} \tilde{\mathrm{F}}
$$

Dove

$$
\widetilde{\mathrm{F}}=\sqrt{\frac{\sin ^{2} \bar{\varphi}}{\mathrm{n}^{2}}+\cos ^{2} \bar{\varphi}}
$$

e infine, invertendo la (80), il modulo del vettore $\tau$.

$$
\tau=\tau_{0}\left(\frac{\mathrm{x}_{\mathrm{p}}}{\mathrm{r}} \tilde{\mathrm{F}}\right)^{\frac{1}{\mathrm{n}+1}}
$$

Le componenti di tensione e dei deformazione risultano quindi:

$$
\begin{aligned}
& \tau_{\mathrm{zy}}=\tau_{0}\left[\frac{\mathrm{x}_{\mathrm{p}}}{\mathrm{r}} \tilde{\mathrm{F}}\right]^{\frac{1}{\mathrm{n}+1}} \cos \bar{\varphi} \\
& \tau_{\mathrm{zx}}=-\tau_{0}\left[\frac{\mathrm{x}_{\mathrm{p}}}{\mathrm{r}} \tilde{\mathrm{F}}\right]^{\frac{1}{\mathrm{n}+1}} \sin \bar{\varphi} \\
& \gamma_{\mathrm{zy}}=\gamma_{0}\left(\frac{\tau}{\tau_{0}}\right)^{\mathrm{n}-1} \frac{\tau_{\mathrm{zy}}}{\tau_{0}}=\gamma_{0}\left(\frac{\mathrm{x}_{\mathrm{p}}}{\mathrm{r}} \tilde{\mathrm{F}}\right)^{\frac{\mathrm{n}}{\mathrm{n}+1}} \cos \bar{\varphi} \\
& \gamma_{\mathrm{zx}}=\gamma_{0}\left(\frac{\tau}{\tau_{0}}\right)^{\mathrm{n}-1} \frac{\tau_{\mathrm{zx}}}{\tau_{0}}=-\gamma_{0}\left(\frac{\mathrm{x}_{\mathrm{p}}}{\mathrm{r}} \widetilde{\mathrm{F}}\right)^{\frac{\mathrm{n}}{\mathrm{n}+1}} \sin \bar{\varphi}
\end{aligned}
$$

E' anche possibile ottenere il legame tra l'angolo $\bar{\varphi}$ nel piano delle tensioni e l'angolo $\varphi$ nel piano fisico, utilizzando le seguenti relazioni:

$$
\frac{\mathrm{y}}{\mathrm{x}}=\frac{\sin \varphi}{\cos \varphi}=\frac{(\mathrm{n}+1) \sin \bar{\varphi} \cos \bar{\varphi}}{\mathrm{n} \cos ^{2} \bar{\varphi}-\sin ^{2} \bar{\varphi}}=\frac{\sin 2 \bar{\varphi}}{\cos 2 \bar{\varphi}+\mathrm{k}}
$$

da cui:

$$
\bar{\varphi}=\frac{\varphi+\arcsin \left(\frac{\mathrm{n}-1}{\mathrm{n}+1} \sin \varphi\right)}{2}
$$

L'intensità dei campi di tensione può essere anche espressa in funzione dell'NSIF elastico, sostituendo esplicitamente $x_{\mathrm{p}}$ : 


$$
\begin{aligned}
& \tau_{\mathrm{zy}}=\left[\frac{\mathrm{n}\left(\mathrm{K}_{3 \rho}^{\mathrm{e}}\right)^{2}}{\pi(\mathrm{n}+1)} \tau_{0}^{\mathrm{n}-1}\right]^{\frac{1}{\mathrm{n}+1}}\left[\frac{\widetilde{\mathrm{F}}}{\mathrm{r}}\right]^{\frac{1}{\mathrm{n}+1}} \cos \bar{\varphi} \\
& \tau_{\mathrm{zx}}=-\left[\frac{\mathrm{n}\left(\mathrm{K}_{3 \rho}^{\mathrm{e}}\right)^{2}}{\pi(\mathrm{n}+1)} \tau_{0}^{\mathrm{n}-1}\right]^{\frac{1}{\mathrm{n}+1}}\left[\frac{\widetilde{\mathrm{F}}}{\mathrm{r}}\right]^{\frac{1}{\mathrm{n}+1}} \sin \bar{\varphi}
\end{aligned}
$$

Alternativamente è anche possibile definire un fattore plastico di intensificazione delle tensioni,

$$
\mathrm{K}_{3 \rho}^{\mathrm{p}}=\lim _{\mathrm{r} \rightarrow \frac{\rho^{+}}{2}} \sqrt{2 \pi} \mathrm{r}^{\frac{1}{\mathrm{n}+1}} \tau_{z y}(\mathrm{r}, \varphi=0)
$$

e riscrivere quindi le tensioni nella forma:

$$
\begin{aligned}
& \tau_{\mathrm{zy}}=\frac{\mathrm{K}_{3 \rho}^{\mathrm{p}}}{\sqrt{2 \pi}}\left(\frac{\widetilde{\mathrm{F}}}{\mathrm{r}}\right)^{\frac{1}{\mathrm{n}+1}} \cos (\bar{\varphi}) \\
& \tau_{\mathrm{zx}}=-\frac{\mathrm{K}_{3 \rho}^{\mathrm{p}}}{\sqrt{2 \pi}}\left(\frac{\widetilde{\mathrm{F}}}{\mathrm{r}}\right)^{\frac{1}{\mathrm{n}+1}} \sin (\bar{\varphi})
\end{aligned}
$$

dove vale la seguente relazione tra NSIF elastico ed NSIF plastico:

$$
\mathrm{K}_{3 \rho}^{\mathrm{p}}=\sqrt{2 \pi}\left[\frac{\mathrm{n}}{\pi(\mathrm{n}+1)} \tau_{0}^{\mathrm{n}-1}\left(\mathrm{~K}_{3 \rho}^{\mathrm{e}}\right)^{2}\right]^{\frac{1}{\mathrm{n}+1}}
$$

Inoltre, usando l'espressione $K_{3 \rho}^{\mathrm{e}}=\tau_{\max }^{\mathrm{e}} \sqrt{\pi \rho}$, le componenti di tensione possono essere riscritte in funzione della tensione massima elastica:

$$
\begin{aligned}
& \tau_{\mathrm{zy}}=\left[\frac{2 \mathrm{n}\left(\tau_{\max }^{\mathrm{e}}\right)^{2}}{(\mathrm{n}+1)} \tau_{0}^{\mathrm{n}-1}\right]^{\frac{1}{\mathrm{n}+1}}\left(\frac{\rho}{2 \mathrm{r}}\right)^{\frac{1}{\mathrm{n}+1}}[\tilde{\mathrm{F}}]^{\frac{1}{\mathrm{n}+1}} \cos \bar{\varphi} \\
& \tau_{\mathrm{zx}}=-\left[\frac{2 \mathrm{n}\left(\tau_{\max }^{\mathrm{e}}\right)^{2}}{(\mathrm{n}+1)} \tau_{0}^{\mathrm{n}-1}\right]^{\frac{1}{\mathrm{n}+1}}\left(\frac{\rho}{2 \mathrm{r}}\right)^{\frac{1}{\mathrm{n}+1}}[\tilde{\mathrm{F}}]^{\frac{1}{\mathrm{n}+1}} \sin \bar{\varphi}
\end{aligned}
$$

All'apice dell'intaglio risulta:

$$
\left.\tau_{\mathrm{zy}}\right|_{\substack{\varphi=0 \\ \mathrm{r}=\mathrm{p} / 2}}=\tau_{\max }^{\mathrm{p}}=\left[\frac{2 \mathrm{n}\left(\tau_{\max }^{\mathrm{e}}\right)^{2}}{(\mathrm{n}+1)} \tau_{0}^{\mathrm{n}-1}\right]^{\frac{1}{\mathrm{n}+1}}
$$

che fornisce un legame analitico tra la massima tensione elastica e plastica.

Usando infine $\tau_{\max }^{\mathrm{p}}$ è possibile riscrivere le tensioni nella seguente forma:

$$
\begin{aligned}
& \tau_{\mathrm{zy}}=\tau_{\max }^{\mathrm{p}}\left(\frac{\rho}{2 \mathrm{r}}\right)^{\frac{1}{\mathrm{n}+1}}[\tilde{\mathrm{F}}]^{\frac{1}{\mathrm{n}+1}} \cos \bar{\varphi} \\
& \tau_{\mathrm{zx}}=-\tau_{\max }^{\mathrm{p}} \frac{1}{\mathrm{n}+1}\left(\frac{\rho}{2 \mathrm{r}}\right)^{\frac{1}{\mathrm{n}+1}}[\tilde{\mathrm{F}}]^{\frac{1}{\mathrm{n}+1}} \sin \bar{\varphi}
\end{aligned}
$$




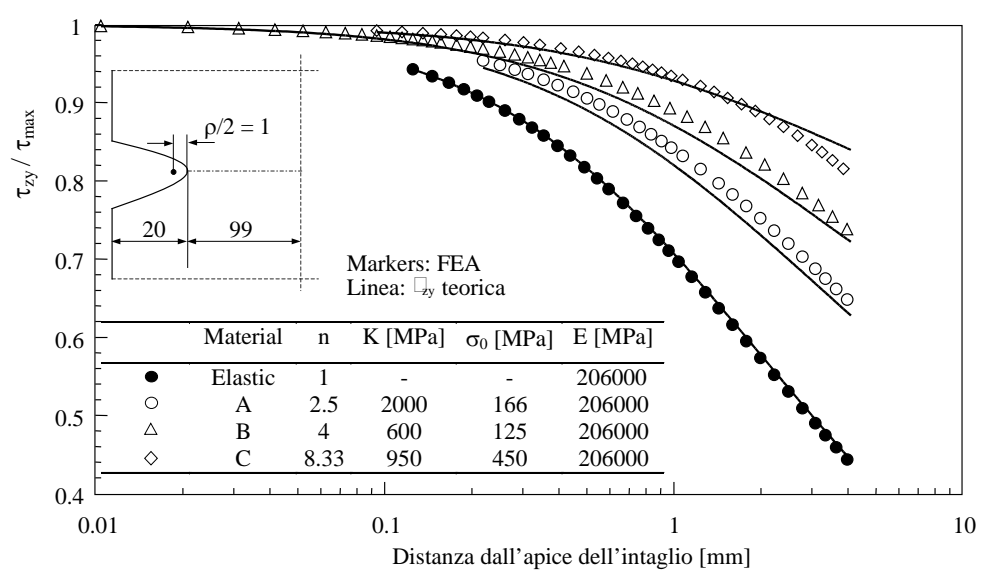

Figura 15: Componenti di tensione $\tau_{z y}$ lungo la bisettrice dell'intaglio per differenti materiali, normalizzate rispetto alla tensione massima sull'apice.

Si noti infine come uguagliando le Eq. 87 e 91, si ottiene la seguente relazione, valida in campo plastico, tra NSIF e tensione massima:

$$
\mathrm{K}_{3 \rho}^{\mathrm{p}}=\tau_{\max }^{\mathrm{p}} \sqrt{2 \pi}\left(\frac{\rho}{2}\right)^{\frac{1}{\mathrm{n}+1}}
$$

La Fig. 15 mostra un confronto tra i risultati analitici e quelli di alcune analisi agli elementi finiti. L'accordo appare ancora soddisfacente.

Confronto con la regola di Neuber. Dall' Eq. 90 è possibile ricavare:

$$
\tau_{\max }^{\mathrm{p}} \cdot \gamma_{\max }^{\mathrm{p}}=\frac{2 \mathrm{n}}{\mathrm{n}+1} \frac{\left(\tau_{\max }^{\mathrm{e}}\right)^{2}}{\mathrm{G}}
$$

Dividendo ambo i membri per $\tau_{\text {nom }} \cdot \gamma_{\text {nom }}=\frac{\tau^{2} \text { nom }}{G}$ si ottiene:

$$
\mathrm{K}_{\tau} \cdot \mathrm{K}_{\gamma}=\frac{2 \mathrm{n}}{\mathrm{n}+1} \mathrm{~K}_{\mathrm{t}}^{2}
$$

diversa dalla proposta di Neuber [14]:

$$
\mathrm{K}_{\tau} \cdot \mathrm{K}_{\gamma}=\mathrm{K}_{\mathrm{t}}^{2}
$$

Tuttavia, tralasciando la traslazione del sistema di riferimento, e quindi assumendo $x_{p}=R_{\mathrm{pz}}$, anzichè $x_{\mathrm{p}}=\frac{2 n}{n+1} R_{\mathrm{pz}}$, si ottiene:

$$
\tau_{\max }^{\mathrm{p}} \cdot \gamma_{\max }^{\mathrm{p}}=\gamma_{0} \tau_{0}\left[\frac{x_{p}}{\mathrm{r}_{0}}\right]=\frac{\left(\tau_{\max }^{\mathrm{e}}\right)^{2}}{\mathrm{G}}
$$

in accordo con Neuber.

Legame con il criterio ESED di Molski e Glinka. In presenza di una condizione di small scale yielding, Molski e Glinka [15] formalizzarono il criterio ESED per intagli raccordati in presenza di sollecitazione di trazione o flessione nella seguente forma: 


$$
\mathrm{W}_{\mathrm{e}}=\mathrm{W}_{\mathrm{p}}
$$

Con l'obiettivo di estendere tale criterio anche a intagli con raggio di raccordo nullo, Lazzarin e Zambardi [16] formularono l'ipotesi che in condizioni di deformazione piana la concentrazione di energia in un volume strutturale che abbracci l'apice dell'intaglio sia costante. Tale volume deve essere completamente immerso in una zona dove i campi di tensione possono essere descritti utilizzando un solo termine nello sviluppo asintotico delle tensioni. In condizioni di small scale yielding, tale ipotesi si traduce nella costanza del valore medio della densità di energia di deformazione

Nel caso di sollecitazioni antiplanari la densità di energia può essere determinate come:

$$
\mathrm{W}=\int_{0}^{\gamma} \tau \mathrm{d} \gamma
$$

Fatte le opportune sostituzioni, l'energia all'apice dell'intaglio in condizioni plastiche risulta:

$$
\mathrm{W}_{\mathrm{p}}=\frac{4 \mathrm{n}^{2}}{(\mathrm{n}+1)^{2}} \frac{\left(\tau_{\max }^{\mathrm{e}}\right)^{2}}{2 \mathrm{G}}-\frac{\mathrm{n}-1}{\mathrm{n}+1} \frac{\tau_{0}^{2}}{2 \mathrm{G}}
$$

Se $n=1$, l'equazione si semplifica in quella valida per il caso lineare elastico:

$$
\mathrm{W}_{\mathrm{e}}=\mathrm{W}_{\mathrm{p}}(\mathrm{n}=1)=\frac{\left(\tau_{\max }^{\mathrm{e}}\right)^{2}}{2 \mathrm{G}}
$$

Quindi:

$$
\frac{\mathrm{W}_{\mathrm{p}}}{\mathrm{W}_{\mathrm{e}}}=\frac{4 \mathrm{n}^{2}}{(\mathrm{n}+1)^{2}}-\frac{\mathrm{n}-1}{\mathrm{n}+1}\left(\frac{\tau_{0}}{\tau_{\max }^{\mathrm{e}}}\right)^{2}
$$

E' chiaro quindi che la costanza dell'energia all'apice dell'intaglio non è verificata.

Consideriamo ora il caso di una cricca e valutiamo l'incremento nel volume strutturale di raggio $R$ dell'energia plastica rispetto a quella lineare elastica:

$$
\begin{aligned}
\mathrm{W}_{\mathrm{P}} & =\tau_{0} \int_{0}^{\gamma}\left(\frac{\gamma}{\gamma_{0}}\right)^{\frac{1}{\mathrm{n}}} \mathrm{d} \gamma=\frac{\mathrm{n}}{\mathrm{n}+1} \frac{\tau^{\mathrm{n}+1}}{\mathrm{G} \tau_{0}^{\mathrm{n}-1}} \\
& =\frac{\mathrm{n}}{\mathrm{n}+1} \frac{1}{\mathrm{G} \tau_{0}^{\mathrm{n}-1}}\left\{\left[\frac{\mathrm{n}\left(\mathrm{K}_{\mathrm{III}}^{\mathrm{e}}\right)^{2}}{\pi(\mathrm{n}+1)} \tau_{0}^{\mathrm{n}-1}\right]^{\frac{1}{\mathrm{n}+1}}\left[\frac{\widetilde{\mathrm{F}}}{\mathrm{r}}\right]^{\frac{1}{\mathrm{n}+1}}\right\}^{\mathrm{n}+1}=\frac{\mathrm{n}^{2} \tilde{\mathrm{F}}}{\pi \mathrm{G}(\mathrm{n}+1)^{2}}\left(\mathrm{~K}_{\text {III }}^{\mathrm{e}}\right)^{2} \mathrm{r}^{-1}
\end{aligned}
$$

Quindi:

$$
\bar{W}_{p}=\frac{E_{p}}{\pi R^{2}}=\frac{2 n^{2}}{\pi^{2} G(n+1)^{2}} \frac{\left(K_{\text {III }}^{e}\right)^{2}}{R} I_{3 p}
$$

dove:

$$
\mathrm{I}_{3 \mathrm{p}}=\int_{0}^{\pi} \tilde{\mathrm{F}} \mathrm{d} \varphi
$$

Quando $\mathrm{n}=1 \mathrm{I}_{3 \mathrm{P}}=\pi$, e quindi: 


$$
\overline{\mathrm{W}}_{\mathrm{e}}=\frac{1}{2 \mathrm{G}} \frac{\left(\mathrm{K}_{\mathrm{III}}^{\mathrm{e}}\right)^{2}}{\pi \mathrm{R}}=\frac{1+v}{\pi \mathrm{E}} \frac{\left(\mathrm{K}_{\mathrm{III}}^{\mathrm{e}}\right)^{2}}{\mathrm{R}}
$$

In conclusione:

$$
\frac{\overline{\mathrm{W}}_{\mathrm{p}}}{\overline{\mathrm{W}}_{\mathrm{e}}}=\frac{4 \mathrm{n}^{2}}{\pi(\mathrm{n}+1)^{2}} \mathrm{I}_{3 \mathrm{p}}
$$

L'equazione (106) fornisce l'incremento nel volume strutturale di raggio R dell'energia plastica rispetto a quella lineare elastica. La Tab. 1 fornisce i valori di $I_{3 \mathrm{P}}$ e $\overline{W_{\mathrm{p}}} / \overline{W_{\mathrm{e}}}$ per alcuni valori dell'indice di incrudimento $n$.

\begin{tabular}{ccc}
\hline $\mathrm{n}$ & $\mathrm{I}_{3 \mathrm{p}}$ & $\frac{\overline{\mathrm{W}}_{\mathrm{p}}}{\overline{\mathrm{W}_{\mathrm{e}}}}$ \\
1 & 3.14159 & 1 \\
2.5 & 2.09436 & 1.36052 \\
4 & 1.7726 & 1.44445 \\
8.33 & 1.44376 & 1.46531 \\
10 & 1.38556 & 1.45797 \\
12 & 1.33475 & 1.44806 \\
\hline
\end{tabular}

Tabella 1: Valori di $\mathrm{I}_{3 \mathrm{p}}$ e $\overline{\mathrm{W}}_{p} / \overline{\mathrm{W}}_{\mathrm{e}}$ ottenuti con un'integrazione numerica dell'Eq.104.

\section{FATTORI PLASTICI DI INTENSIFICAZIONE DELLE TENSIONI PER INTAGLI A SPIGOLO VIVO SOGGETTI A TAGLIO ANTIPLANARE}

$\mathrm{U}$

tilizzando in maniera opportuna le proprietà della trasformazione odografica, Lazzarin e Zappalorto [20] sono stati in grado di determinare la seguente relazione tra NSIF di modo III plastici ed elastici, valida per materiali che presentano un curva tensioni deformazioni conforme a quella rappresentata in Fig. 13:

$$
\mathrm{K}_{3, \mathrm{p}}=\sqrt{2 \pi}\left[-\frac{\mathrm{m}}{\lambda_{3}(1-\mathrm{m})}\left(\frac{\mathrm{K}_{3, \mathrm{e}}}{\sqrt{2 \pi}}\right)^{\frac{1}{1-\lambda_{3}}} \frac{1}{\tau_{0}{ }^{\mathrm{m}+\omega}}\right]^{\frac{1}{1-\mathrm{m}}}
$$

Nella relazione $107 K_{3, \mathrm{e}}$ rappresenta l'NSIF determinato con un'analisi lineare elastica, $\omega$ è un parametro che dipende dall'angolo di apertura, mentre $\mathrm{m}$ dipende sia dall'angolo di apertura che dall'indice di incrudimento. L'Eq. 107 è stata verificata con una serie di analisi agli elementi finiti, mostrando un ottimo accordo in regime di small scale yielding (un esempio è riportato in Fig. 16).

Il frame analitico sviluppato da Lazzarin e Zappalorto ha permesso inoltre di determinare delle espressioni in forma chiusa per la densità di energia di deformazione nel volume di controllo e per il J-integral di Rice in funzione dei fattori plastici di intensificazione delle tensioni:

$$
\begin{aligned}
& \bar{W}_{p}=3^{\frac{n+1}{2}} B_{W}(n, 2 \alpha) \frac{\left(K_{3, p}\right)^{n+1}}{K^{n}} R^{\frac{n+1}{m-1}} \\
& J_{3, p}=3^{\frac{n+1}{2}} B_{J}(2 \alpha, n) \frac{\left(K_{3, p}\right)^{n+1}}{K^{n}} R^{\frac{n+m}{m-1}}
\end{aligned}
$$


Anche per queste relazioni, l'accordo con i risultati di alcune analisi agli elementi finiti si è dimostrato molto soddisfacente (Fig. 17 e 18).

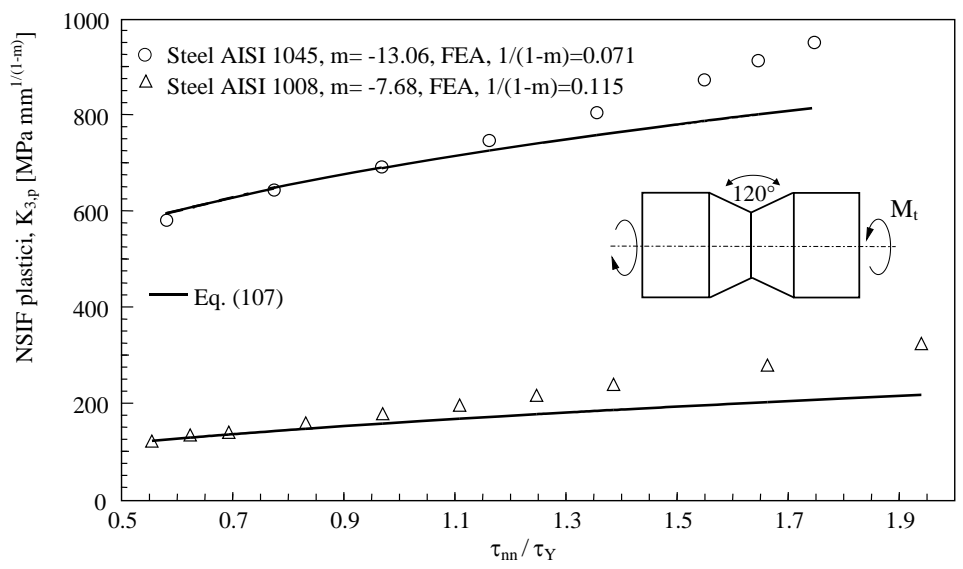

Figura 16: NSIF plastici in accordo con la teoria in confronto con i risultati di analisi agli elementi finiti.

Intaglio a spigolo vivo $\left(2 \alpha=120^{\circ}\right)$.

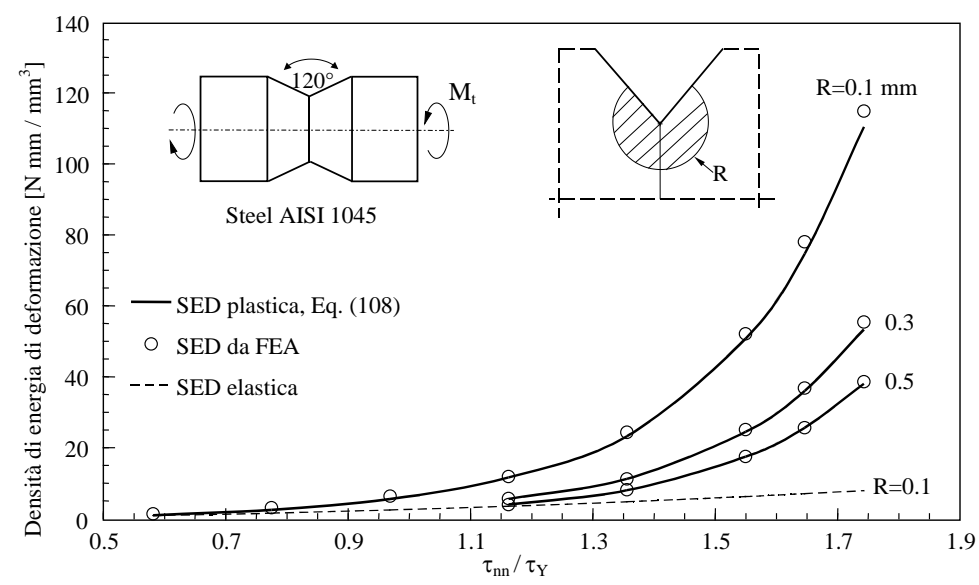

Figura 17: Densità di energia di deformazione in accordo con l' Eq. 108 e confronto con i risultati di alcune analisi agli elementi finiti; intaglio a spigolo vivo $\left(2 \alpha=120^{\circ}\right)$; acciaio AISI 1045.

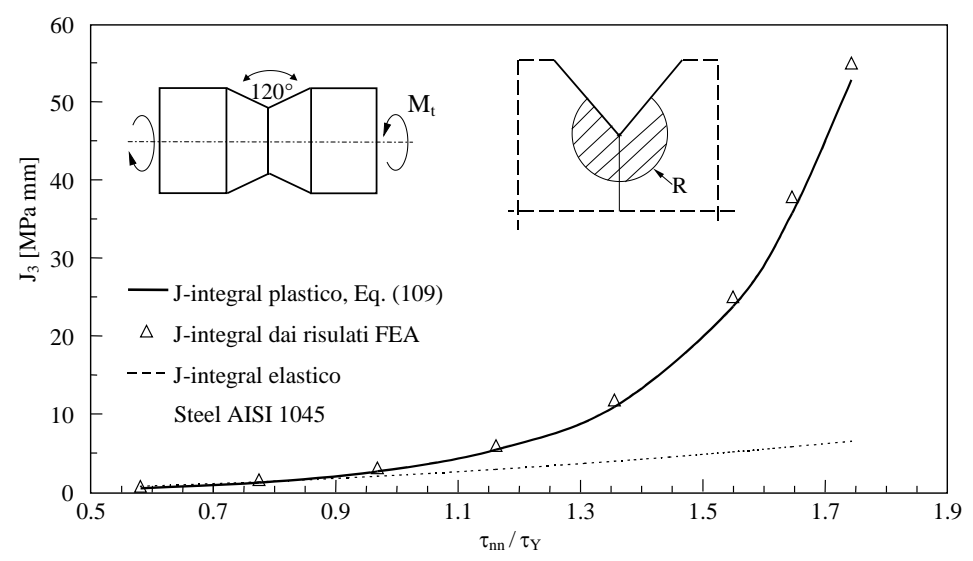

Figura 18: J-integral in accordo con l'Eq.109 e confronto con i risultati di alcune analisi agli elementi finiti; intaglio a spigolo vivo $\left(2 \alpha=120^{\circ}\right)$; acciaio AISI 1045. 


\section{CONCLUSIONI}

$\mathrm{S}$

ono state sviluppate delle equazioni in forma chiusa per descrivere le distribuzioni di tensione generate da intagli di varia forma in alberi intagliati soggetti a torsione, sia in regime lineare elastico sia elastoplastico. Le soluzioni lineari elastiche sono state ottenute utilizzando un approccio basato sull'uso combinato del metodo dei potenziali complessi e di un sistema di coordinate curvilinee. I risultati analitici ottenuti per le componenti di tensione appaiono in soddisfacente accordo con i risultati numerici per un ampio range di forme d'intaglio (dalla cricca all'intaglio circolare), in relazione a corpi assialsimmetrici infiniti e finiti.

Le soluzioni elastoplastiche sono state ottenute invece utilizzando la trasformazione odografica introdotta da Hult e McClinotck e risolvendo il problema di Neumann. Le tensioni sono state espresse dapprima in funzione della massima tensione plastica di taglio presente sull'apice dell'intaglio, poi in funzione di un NSIF plastico generalizzato, valido per un intaglio raccordato, evidenziando analiticamente il legame esistente tra questi due parametri.

E' stata inoltre formalizzata un'espressione in forma chiusa, valida nel campo dello small scale yielding, fra la massima tensione sull'apice dell'intaglio valutata in campo plastico ed il corrispondente valore ottenuto per mezzo di un'analisi lineare elastica.

Infine, per mezzo del frame analitico sviluppato, sono state ridiscusse due regole molto diffuse in letteratura, la regola di Neuber e il criterio ESED di Molski e Glinka, evidenziandone analiticamente limiti e campo di applicabilità.

\section{BIBLIOGRAFIA}

[1] H. Neuber, “Theory of notch stresses", Splinger-Verlag, Berlin (1958).

[2] M. Creager, P.C. Paris, Int. J. Fract. Mech., 3 (1967) 247.

[3] A.Seweryn, K. Molski, Eng. Fract. Mech., 55 (1996) 529.

[4] J. Qian, N. Hasebe, Eng. Fract. Mech., 56 (1997) 729.

[5] M.L. Dunn, W. Suwito, S. Cunningham, Eng. Fract. Mech., 57 (1997) 417.

[6] J.W. Hutchinson, J. Mech. Phys. Solids, 16 (1968a) 13.

[7] J.W. Hutchinson, J. Mech. Phys. Solids, 16 (1968b) 337.

[8] J.R. Rice, G.F. Rosengren, J. Mech. Phys. Solids, 16 (1968) 1.

[9] S.M. Sharma, N. Aravas, J. Mech. Phys. Solids, 39 (1991) 1043.

[10] Z.B. Kuang, X.P. Xu, Int. J. Fract., 35 (1987) 39.

[11] P. Lazzarin, R. Zambardi, P. Livieri, Int. J. Fract. 107 (2001) 361.

[12] J.A.H. Hult, F.A. McClintock, 9th Int Cong Appl Mech (1956), 8, Brussels.

[13] J.R. Rice, J. Appl. Mech., 34 (1967) 287.

[14] H. Neuber, J. Appl. Mech., 28 (1961) 544.

[15] K. Molski, G. Glinka, Mater. Sci. Engng., 50 (1981) 93.

[16] P. Lazzarin, R. Zambardi, Fatigue Fract. Engng. Mater. Struct., 25 (2002) 917.

[17] P.Lazzarin, M. Zappalorto, J.R. Yates, Int. J. Engineering Science, 45 (2007) (2-8), 308.

[18] M. Zappalorto, P. Lazzarin, Int. J. Fract., 148 (2007), 139.

[19] M. Zappalorto, P. Lazzarin, J. Yates, Int. J. Solids Struct., 45(2008) 4879.

[20] Lazzarin P, Zappalorto M. Plastic notch stress intensity factors for pointed V-notches under antiplane shear loading, in stampa su Int. J. Fracture.

[21] L. N. G. Filon, Philosophical transactions of the Royal Society of London, A 193 (1900) 309.

[22] S.P. Timoshenko, J.N. Goodier, “Theory of elasticity 3 ${ }^{\text {rd }}$ Edition”, McGraw-Hill, New York (1970).

[23] N. Hasebe, Y. Kutanda, Eng. Fract. Mech., 10 (1978) 215.

[24] G.R. Irwin, Sagamore Research Conference Proceedings, Vol. 4 (1961), Syracuse University Research Institute, Syracuse NY, 63.

[25] J.D. Unger, “Analytical fracture mechanics" Dover publication (2001).

[26] G. Valiron, "The geometric theory of ordinary differential equations and algebraic functions", Math Science Pr. (1984)

[27] A. R. Forsyth, “A Treatise on Differential Equations”, Dover Publications (1996).

[28] G.P. Cherepanov, J. Appl. Math. Mech., 26 (1962) 1040.

[29] J.W. Hutchinson, "Non linear fracture mechanics", Department of Solid Mechanics, Technical University of Denmark (1979). 


\section{APPENDICE A. DISTRIBUZIONI DI TENSIONE LINEARI ELASTICHE PER UN INTAGLIO SEMI-ELLITTICO CON} UN GENERICO ANGOLO DI INCLINAZIONE $\beta$

$\mathrm{P}$

er semplicità, la trattazione viene limitata solamente al caso di albero infinito. Indicato con $\beta$ il generico angolo di inclinazione dell'intaglio (Fig. A1) è necessario determinare un nuovo sistema di condizioni al contorno. I campi di tensione nel sistema di riferimento cartesiano $(x, y, z)$ sono:

$\begin{aligned} \tau_{z x} & =A_{1}+B_{1} \frac{\sinh 2 \xi}{\cosh 2 \xi-\cos 2 \eta}+B_{2} \frac{\sin 2 \eta}{\cosh 2 \xi-\cos 2 \eta} \\ \tau_{z y} & =-A_{2}-B_{2} \frac{\sinh 2 \xi}{\cosh 2 \xi-\cos 2 \eta}+B_{1} \frac{\sin 2 \eta}{\cosh 2 \xi-\cos 2 \eta}\end{aligned}$

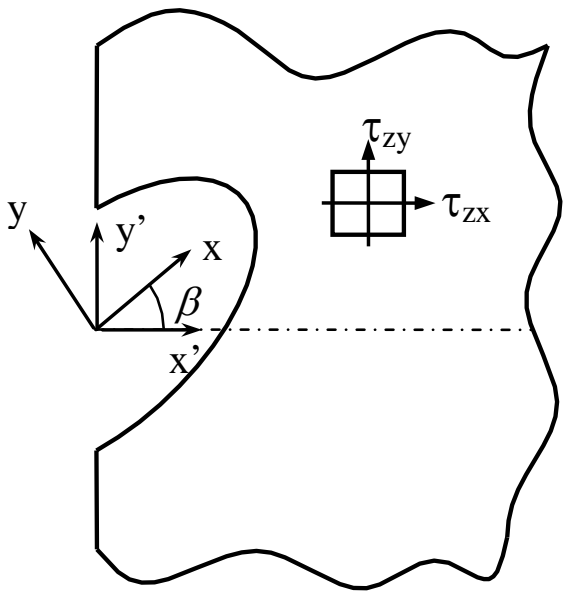

Figura A1: Intaglio ellettico inclinato di un angolo $\beta$ rispetto alla direzione $x^{\prime}$.

Le nuove condizioni al contorno possono quindi essere scritte come:

1. At $|z| \rightarrow \infty ; \tau_{z y^{\prime}}=\tau_{z y, \text { nom }}=\tau$ and $\tau_{z x^{\prime}}=0$

2. $\left.\tau_{z x}\right|_{\xi=\xi_{0}} \prod_{\substack{0 \\ \xi=0}}=0$

3. $\tau_{z y} \mid \eta= \pm \frac{\pi}{2}=0$.

$$
\xi=\xi_{0}
$$

Utilizzando le formule di trasformazione delle tensioni:

$$
\left\{\begin{array}{l}
\tau_{z x^{\prime}} \\
\tau_{z y^{\prime}}
\end{array}\right\}=\left[\begin{array}{cc}
\cos \beta & \sin \beta \\
-\sin \beta & \cos \beta
\end{array}\right]\left\{\begin{array}{c}
\tau_{z x} \\
\tau_{z y}
\end{array}\right\}
$$

È possible riscrivere le condizioni al contorno come:

$$
\left\{\begin{array}{l}
\left(A_{1}+B_{1}\right) \cos \beta-\left(A_{2}+B_{2}\right) \sin \beta=0 \\
-\left(A_{1}+B_{1}\right) \sin \beta-\left(A_{2}+B_{2}\right) \cos \beta=\tau \\
A_{1}+\frac{a}{b} B_{1}=0 \\
A_{2}+B_{2} \frac{b}{a}=0
\end{array}\right.
$$

E la soluzione generale del sistema, in funzione di $\beta$, è la seguente: 


$$
\left\{\begin{array}{l}
A_{1}=-\frac{\tau a}{a-b} \sin \beta \\
A_{2}=\frac{\tau b}{a-b} \cos \beta \\
B_{1}=\frac{\tau b}{a-b} \sin \beta \\
B_{2}=-\frac{\tau a}{a-b} \cos \beta
\end{array}\right.
$$

Le componenti di tensione $\tau_{\mathrm{zy}}$ e $\tau_{\mathrm{zx}}$ ottenute combinando le Eq. A1, A4 sono mostrate in Fig. A2 in funzione della distanza $\mathrm{x}$ dall'apice dell'intaglio, e confrontate con i risultati di un'analisi agli elementi finiti. L'accordo è ancora molto soddisfacente.

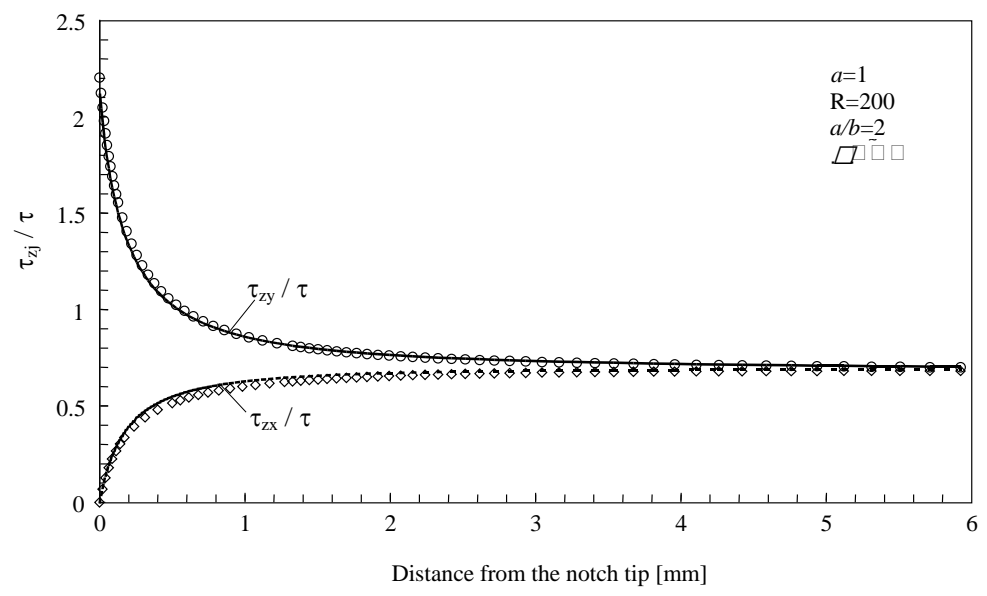

Figura A2: Componenti di tensione $\tau_{z y}$ and $\tau_{\mathrm{zx}}$ lungo la direzione $\eta=0$.

Le tensioni sono normalizzate rispetto alla tensione nominale.

\section{APPENDICE B. UN LEGAME ANALITICO TRA LE DISTRIBUZIONI DI TENSIONE LINEARI ELASTICHE INDOTTE DA INTAGLI DI DIFFERENTE FORMA SOGGETTI A TORSIONE}

$\longrightarrow$

onsideriamo l'espressione della distribuzione di tensione $\tau_{\mathrm{zy}}$ lungo la bisettrice di un intaglio semi-ellittico [17], trascurando il decremento della tensione nominale:

$$
\tau_{\mathrm{zy}}=\frac{\mathrm{b} \tau_{\max }}{\mathrm{a}^{2}-\mathrm{b}^{2}}\left(\frac{\mathrm{ax}}{\sqrt{\mathrm{x}^{2}-\mathrm{c}^{2}}}-\mathrm{b}\right)
$$

Indicando con x' la distanza dalla'apice dell'intaglio, $x^{\prime}=x-a$ e:

$$
\tau_{\text {zy }}=\frac{\mathrm{b} \tau_{\max }}{\mathrm{a}^{2}-\mathrm{b}^{2}}\left(\frac{\mathrm{a}\left(\mathrm{x}^{\prime}+\mathrm{a}\right)}{\sqrt{\left(\mathrm{x}^{\prime}+\mathrm{a}\right)^{2}-\mathrm{c}^{2}}}-\mathrm{b}\right)
$$

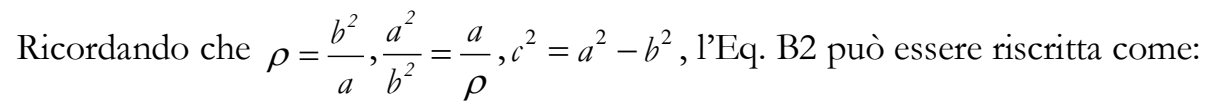




$$
\begin{aligned}
\tau_{z y} & =\frac{b a \tau_{\max }}{a^{2}\left(1-\frac{b^{2}}{a^{2}}\right)}\left(\frac{\left(x^{\prime}+a\right)}{\sqrt{\left(x^{\prime}+a\right)^{2}-c^{2}}}-\frac{b}{a}\right)=\sqrt{\frac{\rho}{a}} \cdot \frac{\tau_{\max }}{\left(1-\frac{\rho}{a}\right)}\left(\frac{\left(x^{\prime}+a\right)}{\sqrt{x^{\prime 2}+2 a x^{\prime}+b^{2}}}-\sqrt{\frac{\rho}{a}}\right) \\
& =\frac{\tau_{\max }}{\left(1-\frac{\rho}{a}\right)}\left(\frac{\frac{x^{\prime}}{a}+1}{\sqrt{\frac{x^{\prime 2}}{a}+2 \frac{x^{\prime}}{\rho}+1}}-\frac{\rho}{a}\right)
\end{aligned}
$$

Ora, se $a>>\rho$ e $x^{\prime}<<a$, l'Eq. B3 tende asintoticamente all'espressione:

$$
\tau_{\mathrm{zy}}=\tau_{\max }\left(\frac{1}{\sqrt{2 \frac{\mathrm{x}^{\prime}}{\rho}+1}}\right)
$$

che rappresenta l'espressione valida per un intaglio parabolico.

Consideriamo ora invece l'espressione della distribuzione di tensione $\tau_{\mathrm{zy}}$ lungo la bisettrice di un intaglio iperbolico [19], trascurando il decremento della tensione nominale:

$$
\left.\tau_{\mathrm{zy}}\right|_{\xi=0}=\frac{\mathrm{b} \tau_{\max }}{\sqrt{\mathrm{c}^{2}-\mathrm{x}^{2}}}
$$

Indicando nuovamente con $x^{\prime}$ la distanza dall'apice dell'intaglio, risulta $x^{\prime}=a-x$, e quindi poichè $c^{2}=a^{2}+b^{2}$, si ottiene:

$$
\tau_{\mathrm{zy}}=\frac{\mathrm{b} \tau_{\max }}{\sqrt{\mathrm{a}^{2}+\mathrm{b}^{2}-\mathrm{x}^{\prime 2}-\mathrm{a}^{2}+2 \mathrm{ax}}}=\frac{\tau_{\max }}{\sqrt{1-\frac{\mathrm{x}^{\prime 2}}{\mathrm{~b}^{2}}+\frac{2 \mathrm{ax}}{\mathrm{b}^{2}}}}
$$

Infine, se $a>>\rho$ e $x^{\prime}<<a$, dato che $\rho=\frac{b^{2}}{a}$, il risultato finale è:

$$
\tau_{\mathrm{zy}}=\tau_{\max }\left(\frac{1}{\sqrt{2 \frac{\mathrm{x}^{\prime}}{\rho}+1}}\right)
$$

di nuovo in accordo con l'espressione valida per un intaglio parabolico. 